\title{
Gradual Typing with Union and Intersection Types
}

\author{
GIUSEPPE CASTAGNA, CNRS - Université Paris Diderot, France \\ VICTOR LANVIN, École Normale Supérieure de Cachan, France
}

\begin{abstract}
We propose a type system for functional languages with gradual types and set-theoretic type connectives and prove its soundness. In particular, we show how to lift the definition of the domain and result type of an application from non-gradual types to gradual ones and likewise for the subtyping relation. We also show that deciding subtyping for gradual types can be reduced in linear time to deciding subtyping for non-gradual types and that the same holds true for all subtyping-related decision problems that must be solved for type inference. More generally, this work not only enriches gradual type systems with unions and intersections and with the type precision that arise from their use, but also proposes and advocates a new style of gradual types programming where union and intersection types are used by programmers to instruct the system to perform fewer dynamic checks.
\end{abstract}

CCS Concepts: • Theory of computation $\rightarrow$ Type theory; $\bullet$ Software and its engineering $\rightarrow$ Functional languages;

Additional Key Words and Phrases: Gradual typing, set-theoretic types, union types, intersection types, negation types

\section{ACM Reference Format:}

Giuseppe Castagna and Victor Lanvin. 2017. Gradual Typing with Union and Intersection Types. Proc. ACM Program. Lang. 1, ICFP, Article 41 (September 2017), 28 pages.

https://doi.org/10.1145/3110285

\section{INTRODUCTION}

A static type system can be an extremely powerful tool for a programmer, providing early error detection, and offering strong compile-time guarantees on the behavior of a program. However, compared to dynamic typing, static typing often comes at the expense of development speed and flexibility, as statically-typed code might be more difficult to adapt to changing requirements. Gradual typing is a recent and promising approach that tries to get the best of both worlds [Siek and Taha 2006]. The idea behind this approach is to integrate an unknown type, usually denoted by "?", which informs the compiler that additional type checks may have to be performed at run time. Therefore, the programmer can gradually add type annotations to a program and controls precisely how much checking is done statically versus dynamically. Gradual typing thus allows the programmer to finely tune the distribution of dynamic and static checking over a program. However, gradualization of single expressions has more limited breadth. We argue that adding full-fledged union and intersection types to a gradual type system makes the transition between dynamic typing and static typing smoother and finer grained, giving even more control to the programmer. In particular, we are interested in developing gradual typing for the semantic subtyping approach [Frisch et al. 2008], where types are interpreted as sets of values. In this approach union

This work is licensed under a Creative Commons Attribution 4.0 International License.

(c) 2017 Copyright held by the owner/author(s).

2475-1421/2017/9-ART41

https://doi.org/10.1145/3110285

Proc. ACM Program. Lang., Vol. 1, No. ICFP, Article 41. Publication date: September 2017. 
and intersection types are naturally interpreted as the corresponding set-theoretic operations, and the subtyping relation is defined as set-containment, whence the name of set-theoretic types. This yields an intuitive and powerful type system in which several important constructions eg, branching, pattern-matching, and overloading - can be typed very precisely. Set-theoretic types, however, exacerbate the shortcomings of static typing. In particular, type reconstruction for intersection type systems is, in general, undecidable. The consequence is that programmers have to add complete type annotations for every variable, which may hinder their development speed; all the more so given that union and intersection type annotations can be syntactically heavy. Adding gradual typing to set-theoretic types may help to alleviate this issue by providing a way to relax the rigidity of certain type annotations via the addition of a touch of dynamic typing, while keeping the full power of static types for critical parts of code.

We said that adding set-theoretic types to a gradual type system makes the transition between dynamic typing and static typing smoother. This is for example the case for function parameters that are to be bound to values of basic types: in the current setting, the only way to gradualize their type is to use "?", while with union and intersection types more precise gradualizations become possible. We illustrate this fact in an ML-like language by progressively refining the following example that we borrow from Siek and Vachharajani [2008].

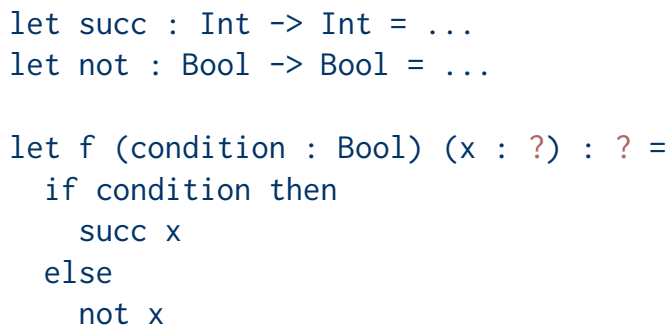

This example cannot be typed using only simple types: the type of $x$ as well as the return type of $f$ change depending on the value of condition. However, this piece of code is perfectly valid in a gradual type system, the compiler will simply add dynamic checks to ensure that the value bound to $x$ can be passed as an argument to succ or not according to the case. Moreover, it will also add checks to ensure that the value returned by $f$ is used correctly. Nevertheless, there are some flaws in this piece of code. For example, it is possible to pass a value of any type as the second argument of $f$ (the type system ensures that the first argument will always be a Boolean). In particular, if one applies the function $f$ to (a Boolean and) a value of type string, then the application will always fail, independently from the value of condition, and despite the fact that the application is statically considered well-typed. This problem can be avoided by set-theoretic types, in particular by using the union type Int | Bool to type the parameter $x$ of the function so as to ensure that every second argument of $f$ that is neither an integer nor a Boolean will be statically rejected by the type checker. This is obtained by the following code

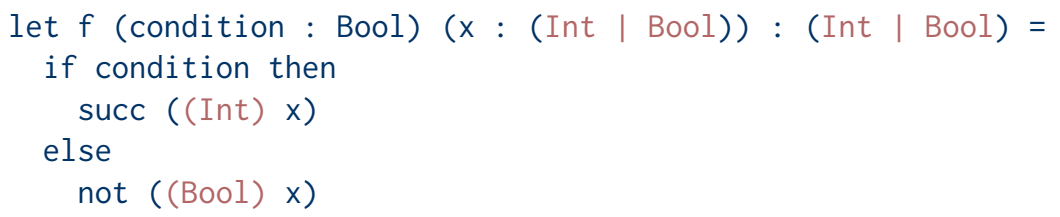

The code above shows that the use of union types fixes the shortcoming we pointed out. However in order to ensure that the applications of succ and not are both well typed we also had to add 
two type casts ${ }^{1}$ that check at run-time whether the argument has the required type and raise an exception otherwise.

REMARK. The use of explicit type-casts should not surprise the reader. Any language with union types needs an operation to dynamically check the type of values that are given union types. Such operations may have different forms and be implemented in different ways and at different degrees: they range from the simple check of constructors/tags (eg, when unions are restricted to datatypes/variant-types), to value inspection primitives such as those of Typed Racket [TobinHochstadt and Felleisen 2008] (eg, procedure?, number?, etc.), till the unconstrained check of the type of a value returned by an expression $e$, that is,

$$
\text { match } e \text { with } t_{1} \rightarrow e_{1}|\ldots| t_{n} \rightarrow e_{n}
$$

which is used in languages like CDuce [Benzaken et al. 2003] where unions are full fledged. ${ }^{2}$ This article studies the latter case and therefore a type cast $(t) e$ is just syntactic sugar for ${ }^{3}$

$$
\text { match } e \text { with } t \rightarrow e||_{-} \rightarrow \text { raise "Cast error". }
$$

In this second definition of $f$ we have ensured, thanks to union types, that every application of $f$ has now a chance to succeed. However, this is obtained at the expenses of the programmer who has now the burden to insert in the code the type-cases/type-casts necessary to ensure safety (in the sense established by Wright and Felleisen [1994]). By using set-theoretic types in conjunction with gradual types, it is possible both to ensure that $f$ will only be applied to booleans or integers and to delegate the insertion of type casts to the system. This is shown by the following piece of code that our system will compile into the previous one.

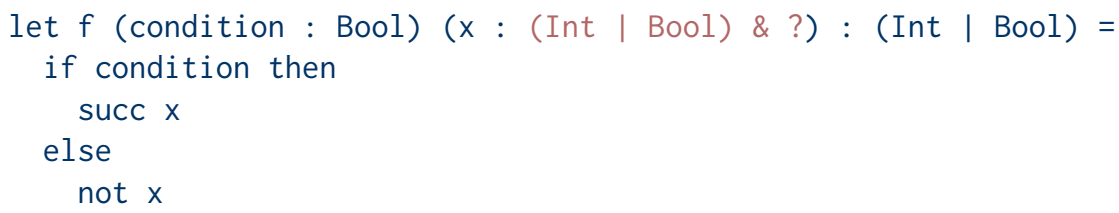

In this example, the variable $x$ is of type ((Int | Bool) \& ?), where " $\&$ " denotes an intersection. This indicates that $x$ has both type (Int | Bool) and type ?. Intuitively, this means that the function $f$ accepts as a second argument a value of any type (which is indicated by ?), as long as this value is also an integer or a Boolean. The effect of having added "\& ?" in the type of the parameter is that the programmer is no longer required to add the explicit casts in the body of the function: the system will take care of it at compile time. The combination of a union type with "\& ?" to type a parameter corresponds to a programming style in which the programmer asks the system to statically enforce that the function will be applied only to arguments in the union type and delegates to the system the dynamic checking, where/if necessary, for each case in the union; and while adding explicit casts in a five-line example such as the above is quite straightforward, in

\footnotetext{
${ }^{1}$ Although we used a C-like syntax these type casts do not perform any type conversion or promotion: they are just dynamic type-checks.

${ }^{2}$ The cost of run-time "deep" type-cases is frequently misunderstood and overestimated. This is occasionally expressed by the belief that having only a restricted set of type inspection primitives would greatly improve runtime performance in terms of time and/or space. Frisch [2004] shows that since most of the values already encode their type information, then in strict languages the overhead of deep dynamic type-cases can be made small: lambda-abstractions must keep their type annotations at runtime but no other subexpression needs to be type-decorated (though in some languages one might need to differentiate constants of different types, e.g. booleans from integers in OCaml). Unconstrained dynamic type-check can then be implemented very efficiently by using the static type information available at compile time (Frisch [2004] proves that it is possible to implement it optimally for every given exploration strategy for values): in practice, it often turns out to be a check of the topmost constructor of a value.

${ }^{3}$ A proper definition is match $e$ with $t \& \mathrm{x} \rightarrow \mathrm{x} \mid{ }_{-} \rightarrow$ raise "Cast error" where $e$ is evaluated just once.
} 
general (eg, in thousand-line modules), it is not always so, whence the interest of having a system that adds all and only the casts that are necessary to ensure type safety. Finally, note that the return type of $f$ is no longer gradual (as it was in the first definition), since union types allow us to define it without any loss of precision. This allows the system to statically reject all cases in which the value expected from $f$ is neither an integer nor a Boolean and which, with the first definition, would be detected only at run-time.

In all the examples above the return type of the function $f$ can be easily and automatically deduced and could, therefore, be omitted. But there are cases in which providing the return type of a function allows the system to deduce a better type and, thus, accept more programs. This is particularly true in conjunction with intersection types, since they allow the programmer to specify different return types for different argument configurations. Consider again the function $f$ above. Since it always returns either an integer or a Boolean, we used as return type (Int | Bool). By using an intersection type it is possible to give $f$ a more precise type in which the return type of $f$ depends on the type of $\mathrm{x}$ :

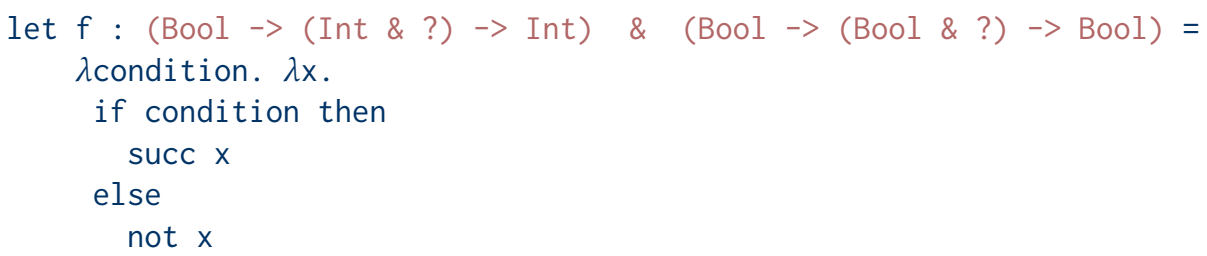

This time, in the body of $f$, the variable $x$ has type (Int \& ?) | (Bool \& ?). This type is equivalent ${ }^{4}$ to ( (Int $\mid$ Bool) \& ?). Hence, the function can be defined with the same body as before, and it accepts as arguments the same values. However, the return type of $f$ now directly depends on the type of $x$ (more precisely, on the type of the value bound to $\mathrm{x}$ ): if it is of type Int, then the function necessarily returns an integer (that is, if the application does not fail), and the same goes for an argument of type Bool.

Having a return type that depends on the type of the input is reminiscent of the typing of overloaded functions (also known as "ad hoc polymorphism"). This correspondence is indeed a strong one, since intersections of arrow types can be used to type overloaded functions (eg, see Benzaken et al. [2003]; Castagna et al. [1995]; see also Forsythe [Reynolds 1996] which uses a limited form of overloading known as coherent overloading). As a matter of fact, our function $f$ is just a curried function that when applied to a Boolean argument returns an overloaded function. This can better be seen by considering type equivalences: the type we declared above for $f$

$$
\text { (Bool -> (Int \& ?) -> Int) \& (Bool -> (Bool \& ?) -> Bool) }
$$

is equivalent to the type

$$
\text { Bool -> ( ((Int \& ?) -> Int) \& ((Bool \& ?) ->Bool) ) }
$$

Therefore an equivalent way to define $f$ would have been

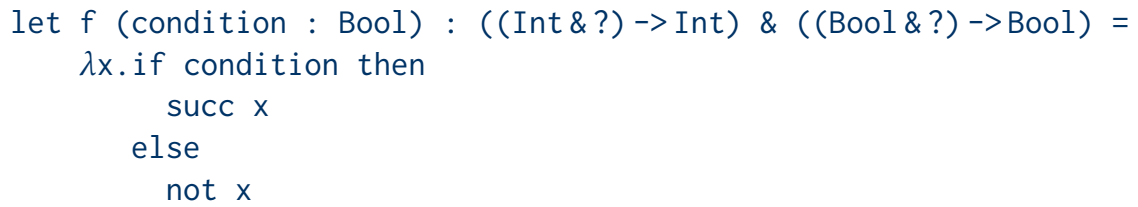

\footnotetext{
${ }^{4}$ Although we did not formally define any equivalence relation, the reader will easily recognize here a classic distributivity rule of set-theoretic unions and intersections. Slightly more formally, in this introduction we consider two types to be equivalent if they are both one subtype of the other (according to Frisch et al. [2008]) and where "?" is considered as some distinguished base type that intersects all the other types.
} 
which shows in a clear way that the application of $f$ to a (necessarily Boolean) argument returns an overloaded function whose result type depends on the type of its argument: the two occurrences of "?" in the input types of the overloaded function indicate that, in both cases (ie, whatever the type of the argument is), some dynamic cast may be needed in the body of the function and, thus, may have to be added at compile-time.

We want to conclude this introduction by mentioning singleton types, that we do not consider in this work but that can be straightforwardly added to our theory without any further modification. In a language such as $\mathbb{C D u c e}$ every value also denotes the singleton type that contains only that value. In particular $\mathbb{C D u c e}$ features two separate types for true and false and the type Bool is then simply defined as the union true | false. In this language, it is then possible to define the most precise type for $f$ without using neither gradual types nor explicit type casts: ${ }^{5}$

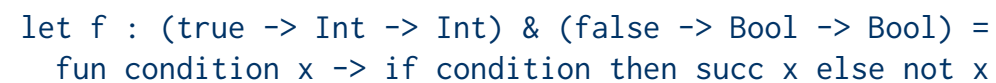

Likewise, in Typed Racket, a similar typing is obtained by "case->", which provides a limited form of intersection:

\section{(: $f$ : (case-> (-> True Integer Integer) ( $->$ False Boolean Boolean))) \\ (define ( $f$ condition $x$ ) (if condition (add1 $x$ ) (not $x)$ ))}

with the caveat that partial applications are not allowed. Of course this works in both cases only because Bool is a finite type, but in general it is not possible to replace gradual types or (equivalently) explicit type casts by using singleton types.

\subsection{Overview}

The examples given so far provide a brief outline of the characteristics of the system we are going to study. In a nutshell, this work develops a theory for gradual set-theoretic types, that is, types that besides the usual type constructors - eg, arrows, products, integers, ...- include a gradual "?" basic type and set-theoretic type connectives: union, intersection, and negation (in the set-theoretic type approach negations are indissociable from unions and intersections). This amounts to defining and deciding their subtyping relation, using them to type a core functional language, and defining a compilation scheme that inserts all and only the type casts required to ensure that every non-diverging well-typed expression will either return a value or raise a cast error. To that end, we proceed as follows. In Section 2, we define the syntax and semantics of the types we are interested in. In particular, we use abstract interpretation [Cousot and Cousot 1977] to define the semantics of our gradual types, following a technique introduced by Garcia et al. [2016]. Section 3 presents a gradually-typed language and its associated type system. This language is an explicitly-typed lambda calculus with a typecase, the latter included to fully exploit set-theoretic types. Section 4 presents the target language, a gradually-typed lambda calculus with explicit casts. In particular, we define its type system and operational semantics and prove its soundness. Finally, section 5 describes a compilation procedure that automatically inserts casts guaranteeing that every well-typed term of our gradually typed language is compiled into a term that if it converges, then it reduces either to a value or to a cast error. A conclusion with directions for future work ends this presentation. For space reasons, lemmas, proofs, and a few definitions are provided as supplemental material in an appendix available on-line.

\footnotetext{
${ }^{5}$ In CDuce "if $e$ then $e_{1}$ else $e_{2}$ " is just syntactic sugar for "match $e$ with true $\rightarrow e_{1} \mid$ false $\rightarrow e_{2}$ ". The current public release of $\mathbb{C D u c e}$ requires all function parameters, such as condition and $x$, to be explicitly typed and, thus, it does not accept the code below. The code below is executable on a experimental prototype version (available at http://www.cduce.org/ocaml/bi) based on the work by Castagna et al. [2016] and that uses a bi-directional type system to infer the types of the parameters of functions.
} 


\subsection{Contributions}

The main contribution of this work is the definition of the static and the dynamic semantics of a language with gradual types and set-theoretic type connectives and the proof of its soundness. In particular, we show how to lift the definition of domain and result type of an application from set-theoretic types to gradual types and likewise for the subtyping relation. We also show that deciding subtyping for gradual types can be reduced in linear time to deciding subtyping on settheoretic types and that the same holds true for all subtyping-related decision problems needed for type inference (notably, computing domains and result types). More generally, this work not only enriches gradual type systems with unions and intersections and with the type precision that arises from their use, but also proposes and advocates a new style of programming with gradual types where union and intersection types are used by the programmer to instruct the system to perform fewer dynamic checks.

\subsection{Related Work}

Our work combines set-theoretic types with gradual typing. The part on set-theoretic types is based on the semantic subtyping framework, as presented by Frisch et al. [2008], while for what concerns the addition of gradual typing we followed and adapted the technique based on abstract interpretation by Garcia et al. [2016] called "Abstracting Gradual Typing” (AGT). However, due to the specific needs of our type system and the difficulty of finding a suitable Galois connection (a standard construction for abstract interpretation), we could apply directly the AGT approach only to "lift" subtyping. In particular, the approach proposed by Garcia et al. [2016] focuses on consistent subtyping, whereas dealing with set-theoretic types requires more precise properties -most notably for lemmas related to term substitution- hence the need for new operators and for a specific dynamic semantics.

There exist other attempts at integrating gradual typing with union and/or intersection types, but none is as general as the one presented here, insofar as they just consider either a partial set of type connectives or limited forms thereof. Siek and Tobin-Hochstadt [2016] study gradual typing for a language with type-case, union types, and recursive types. Intersection and negation types are not considered and union types are in a restricted version, since it is not possible to form the union of any two types but just of types having different top-most constructors: so for instance it is not possible to union two arrows. This limitation is reflected in the type-case expression which can only check the topmost constructor of a value (eg, integer, product, arrow, ...) but not its type. The different cases of the type-case construction of Siek and Tobin-Hochstadt [2016] are functions that, if selected, are applied to the matched value; this allows a form of occurrence typing [Tobin-Hochstadt and Felleisen 2008]. Our type-case is more general than the one by Siek and Tobin-Hochstadt [2016] since expressions can be checked against any type, and occurrence typing can be encoded (see Footnote 13 later on). For the sake of simplicity we considered neither product nor recursive types (though all their theory is already developed by Frisch et al. [2008]) and disregarded blame, but otherwise our work subsumes the one by Siek and Tobin-Hochstadt [2016].

Jafery and Dunfield [2017] present a type system that contains both refinement sums and gradual sums. Similarly to our approach, they define a gradually-typed source language and a type-directed translation to a target language that contains casts. However, their approach is very different from ours: their sums are disjoint unions in which elements are explicitly injected by a constructor; as such, their sums do not have the set-theoretic property of unions (eg, they are neither idempotent, nor commutative, nor satisfy usual distribution laws). Also, gradual typing is confined to sum types, since the motivation of their work is to allow the programmer to gradually add refinements that make the enforcement of exhaustive pattern matching possible, a problem that does not subsists in 
our work or, more generally, in languages with set-theoretic types where exhaustiveness of pattern matching is easily verifiable. ${ }^{6}$ Finally, Jafery and Dunfield [2017] leave intersection types as future work.

Our approach also relates to a recent work by Lehmann and Tanter [2017], which presents a way to combine gradual typing with full-fledged refinement types, where formulae can contain unions, intersections, and negations. They encounter problems similar to ours, most notably when trying to find a suitable Galois connection to use for AGT. However, our work focuses on set-theoretic types which behave differently from refinement types, in particular when it comes to subtyping, function types, or when evaluating casts.

For what concerns programming languages, there have been few attempts at providing a language with union and intersection type connectives and some form of gradual typing, whether it be via generics or using a specific unknown type. Facebook's Flow [Chaudhuri 2014] is an impressive gradually-typed version of JavaScript that provides union and intersection types, occurrence typing, as well as a type "any" that behaves as our unknown type "?": however any is used in Flow only to shunt off the type system, but not to insert casts. By inserting by hand appropriate type annotations and casts it is possible to make the examples of our introduction type-check in Flow. Therefore, it would be interesting to see how to use our system to give the programmer the option to let Flow add the necessary casts and annotations. Also, even though an implementation is available, there is no detailed (let alone formal) definition of Flow's type system: our work certainly provides a good starting point for it. Typed Racket is another example that provides sound gradual typing (in a coarser-grained form than the one obtained by using explicit gradual types) as well as true union types. It does not support full intersection types (just the limited form we showed to be provided by the case-> construct in which types are considered in order from the first to the last), it includes recursive types and polymorphic function, performs a limited form of type reconstruction, and, above all, is a full-fledged programming language. It also features occurrence typing [TobinHochstadt and Felleisen 2008], which refines the types of some variable depending on the result of a conditional, and that can be encoded by our typecase construct. As it is the case for Flow, in Typed Racket the examples of our introduction are accepted only after the insertion of explicit type inspection primitives by the programmer. Contrary to Flow, however, using our system to insert these casts in Typed Racket does not seem appropriate, both for the absence in Typed Racket of an explicit "unknown" type and, more generally, for the general spirit of the approach.

\section{TYPES}

In this section, we define the syntax of set-theoretic types (that in this work we call "static types") and extend them to obtain gradual types. We define the semantics of the latter in terms of the former. This semantic is then used to lift to gradual types relevant definitions given for set-theoretic types, notably the subtyping relation.

\subsection{Type Syntax}

Definition 1. (Types) The set STypes of static types and the set GTypes of gradual types are inductively generated by the following grammars:

$$
\begin{gathered}
t \in \text { STypes }::=b|t \rightarrow t| t \vee t|t \wedge t| \neg t|\mathbb{O}| \mathbb{1} \\
\tau \in \text { GTypes }::=?|b| \tau \rightarrow \tau|\tau \vee \tau| \tau \wedge \tau|\neg t| \mathbb{O} \mid \mathbb{1}
\end{gathered}
$$

\footnotetext{
${ }^{6}$ This is due to the specific definition of set-theoretic patterns which combine singleton types and set-theoretic connectives in such a way that checking exhaustiveness amounts to checking a subtyping relation: see, for instance, [Castagna et al 2016, sect. 4.2].
} 
where $b$ ranges over the set of base types (eg, Int, Bool, ...). We also single out the atomic (gradual) types as follows:

$$
a \in \text { Atom }::=b \mid t \rightarrow t \quad \alpha \in \text { GAtom }::=?|b| \tau \rightarrow \tau
$$

The set STypes of static types, ranged over by $s, t, \ldots$, is formed by basic types, the type constructor " $\rightarrow$ " for function types, type connectives for union, intersection and negation types, as well as $\mathbb{D}$ and $\mathbb{1}$ which denote respectively the bottom and the top type. The set GTypes of gradual types, ranged over by $\sigma, \tau, \ldots$, is obtained by adding to the static types the unknown type "?", which stands for the absence of type information (not to be confused with $\mathbb{0}$ or $\mathbb{1}$ ). As anticipated, we also included negation types, which correspond to the set-theoretic complement (ie, a well-typed expression has type $\neg t$ if and only if it does not have type $t$ ). These play an important role in our theory insofar as all subtyping-related algorithms as well as the type-inference of type-case expressions fundamentally rely on them. ${ }^{7}$ Notice however that we do not allow negations of gradual types. (the reason is that we could neither conceive any reasonable interpretation for a type such as " $\neg$ ?", nor we were able to deduce it from our formalization). We use the standard convention that connectives ( ie, $\wedge, \vee$, and $\neg$ ) are given a higher precedence than constructors $(i e, \rightarrow)$. We also use the symbol "I" to denote the difference of a (possibly gradual) type with another, this being defined in the usual set-theoretic way, that is, $\tau \backslash t \stackrel{\text { def }}{=} \tau \wedge \neg t$.

We suppose that static types come equipped with the subtyping relation $\leq$ defined by Frisch et al. [2008] (our static types are a strict subset of those defined in [Frisch et al. 2008]). As hinted in the introduction, this subtyping relation is defined by interpreting static types as sets of values (ie, either constants or $\lambda$-abstractions) that have that type, and then defining subtyping as set containment (ie, a static type $s$ is a subtype of a static type $t$ if and only if $t$ contains all the values of type $s$ ). More precisely: Int is the set of all integers; Bool is the set $\{$ true, false $\} ; \mathbb{D}$ is the empty set; $\mathbb{1}$ is the set of all (well-typed) values; type connectives are interpreted as the corresponding set-theoretic operators (eg, $s \vee t$ is the union of the values of the two types, while $\neg t$ is the set of all values that are not in/of type $t$ ); $s \rightarrow t$ is set of all $\lambda$-abstractions that when applied to a value of type $s$ return only results of type $t$. In particular, $\mathbb{O} \rightarrow \mathbb{1}$ is the set of all functions ${ }^{8}$, which is why we call every subtype of $\mathbb{O} \rightarrow \mathbb{1}$ a function type. We use $\simeq$ to denote the equivalence relation induced by the subtyping relation (ie, $t_{1} \simeq t_{2}$ if and only if $t_{1} \leq t_{2}$ and $t_{2} \leq t_{1}$ ): intuitively, two static types are equivalent if and only if they denote the same set of values.

In the Introduction we described the intuitive semantics of gradual types. In the next section we formalize this intuition. But before that let us stress again that? must not be confused with $\mathbb{O}$ or $\mathbb{1}$ : for instance, a function of type $? \rightarrow$ Int can take an argument of a certain unknown type and return an integer, but, contrary to $\mathbb{1} \rightarrow$ Int, this application might fail if the unknown type turns out at runtime not to be a super-type of the type of the argument; likewise, while the application of a function in Int $\rightarrow$ ? to an integer may return some result (whose type is unknown), the application to an integer of a function in Int $\rightarrow \mathbb{O}$ always diverges (since, if it converged, then it ought to return a value belonging to the empty type, which is impossible).

\subsection{Semantics of Types}

Since STypes $\subset$ GTypes, then any definition on gradual types can be implicitly restricted to static types. However, this containment is strict and, thus, the converse is not so straightforward. In the rest of this section we present a way to do this reverse operation, namely, to lift the definitions of relations (foremost, subtyping) and operators (eg, domain and result type) given on static types to

\footnotetext{
${ }^{7}$ The use of negation types becomes interesting for the programmer mainly in the presence of polymorphism $-c f$. [Castagna et al. 2015, 2014] - which is why in this work we put the emphasis essentially on unions and intersections.

${ }^{8}$ Actually, for every type $t$, all types of the form $\mathbb{D} \rightarrow t$ are equivalent and each of them denotes the set of all functions.
} 
gradual types. Our approach uses abstract interpretation [Cousot and Cousot 1977] to interpret gradual types as sets of static types, as first proposed by Garcia et al. [2016].

Concretization. The first step in lifting the semantics of static types to gradual types is to define the concretization of a gradual type as a set of static types. This is based on the intuition that the gradual type ? can turn out to be any type, that is, the set of its possible interpretations is STypes. Formally, we define the concretization function $\gamma$ such that $\gamma(\tau)$ returns the set of static types obtained by replacing each occurrence of ? in $\tau$ by some static type.

Definition 2. (Concretization) The concretization function $\gamma:$ GTypes $\rightarrow \mathscr{P}$ (STypes) is defined as follows:

$$
\begin{aligned}
\gamma(?) & =\text { STypes } & \gamma\left(\tau_{1} \rightarrow \tau_{2}\right) & =\left\{t_{1} \rightarrow t_{2} \mid t_{i} \in \gamma\left(\tau_{i}\right)\right\} \\
\gamma\left(\tau_{1} \vee \tau_{2}\right) & =\left\{t_{1} \vee t_{2} \mid t_{i} \in \gamma\left(\tau_{i}\right)\right\} & \gamma(b) & =\{b\} \\
\gamma\left(\tau_{1} \wedge \tau_{2}\right) & =\left\{t_{1} \wedge t_{2} \mid t_{i} \in \gamma\left(\tau_{i}\right)\right\} & \gamma(\mathbb{O}) & =\{\mathbb{0}\} \\
\gamma(\neg t) & =\{\neg t\} & \gamma(\mathbb{1}) & =\{\mathbb{1}\}
\end{aligned}
$$

In our set-theoretic framework, the concretization of a gradual type has several interesting properties. In particular, for every gradual type $\tau$, the set of its concretizations $\gamma(\tau)$ has a maximum and a minimum with respect to the subtyping relation $\leq$. That is, $(\gamma(\tau), \leq)$ is a closed sublattice of (STypes, $\leq$ ). This is a direct consequence of the fact that the set of static set-theoretic types is a complete lattice, bounded by $\mathbb{1}$ and $\mathbb{D}$.

Proposition 1. (Gradual Extrema) For every gradual type $\tau \in$ GTypes, there exist in $\gamma(\tau)$ two static types, noted $\tau^{\Uparrow}$ and $\tau^{\Downarrow}$, such that for every type $t \in \gamma(\tau), \tau^{\Downarrow} \leq t \leq \tau^{\Uparrow}$.

Moreover, $\tau^{\Uparrow}$ (resp. $\tau^{\Downarrow}$ ) is obtained from $\tau$ by replacing all covariant occurrences of ? by $\mathbb{1}$ (resp. $\mathbb{0}$ ) and all contravariant occurrences of ? by $\mathbb{0}($ resp. $\mathbb{1})$.

Subtyping. Subtyping is a binary relation on static types. We want to define a conservative extension of this predicate to gradual types. More generally, given any predicate $P\left(t_{1}, \cdots, t_{n}\right)$ on static types, we want to define a predicate $\widetilde{P}\left(\tau_{1}, \cdots, \tau_{n}\right)$ on gradual types such that $\widetilde{P}$ coincides with $P$ on static types (conservative extension) and encompasses the intuition of gradual typing. This is done by using the concretization function as follows:

Definition 3. (Predicate lifting [Garcia et al. 2016]) For every predicate $P \in$ STypes $^{n}$, we define its consistent lifting $\widetilde{P} \in$ GTypes $^{n}$ as:

$$
\widetilde{P}\left(\tau_{1}, \cdots, \tau_{n}\right) \stackrel{\text { def }}{\Longleftrightarrow} \exists\left(t_{1}, \cdots, t_{n}\right) \in \gamma\left(\tau_{1}\right) \times \cdots \times \gamma\left(\tau_{n}\right) \quad \text { s.t. } \quad P\left(t_{1}, \cdots, t_{n}\right)
$$

Notice that, since $\gamma(t)=\{t\}$ for every static type $t$, then $\widetilde{P}$ coincides with $P$ on static types.

Intuitively, a predicate is true for some gradual type if and only if the static counterpart of the predicate is true for some particular concretization of these types. If we apply lifting to the subtyping relation, we obtain that, for every pair of gradual types $\sigma$ and $\tau, \sigma \widetilde{\leq} \tau$ if and only if there exist two types $(s, t) \in \gamma(\sigma) \times \gamma(\tau)$ such that $s \leq t$ (the relation $\widetilde{\leq}$ is usually referred to as "consistent subtyping"). However, this definition can be simplified using the fact, stated by Proposition 1, that $(\gamma(\tau), \leq)$ is a sublattice of (STypes, $\leq$ ).

Proposition 2. (Consistent subtyping) For every pair of gradual types $\sigma, \tau$, the following equivalence holds:

$$
\sigma \widetilde{\leq} \tau \Longleftrightarrow \sigma^{\Downarrow} \leq \tau^{\Uparrow}
$$

This proposition is important since it implies that the subtyping problem for gradual types can be linearly reduced (as explained in Proposition 1) to the same problem on static types and, thus, that we can re-use the algorithms that already exist for static types. In particular, since $\tau^{\Uparrow}$ and $\tau^{\Downarrow}$ are of 
the same size as $\tau$, then the decision problems of $\widetilde{\leq}$ and $\leq$ have the same algorithmic complexity. This property also illustrates the (well-known) fact that consistent subtyping $\widetilde{\leq}$ is not transitive. Indeed, ? is a consistent subtype of every type (as $?^{\Downarrow}=\mathbb{O}$ ) but also a consistent super-type of every type $\left(\right.$ as $\left.?^{\Uparrow}=\mathbb{1}\right)$. Thus, if consistent subtyping were to be transitive, it would collapse to the trivial full relation where $\sigma \widetilde{\leq} \tau$ for every gradual types $\sigma$ and $\tau$. We think that gradual quasi-subtyping would have been more appropriate a name for this relation, but we preferred to adopt the accepted terminology.

\subsection{Operators on Types}

Lifting subtyping from static to gradual types was easy: we just adapted standard techniques of abstract interpretation on the lines of Garcia et al. [2016]. Lifting type operators, instead, is far more difficult and constitutes one of the hardest technical achievements of our work. Operators on types are necessary to define type inference. In particular, in order to type any functional language (notably, to type its applications) one needs at least two operators: (i) the $\operatorname{dom}($.) operator that given a function type $t$ (ie, a subtype of $\mathbb{0} \rightarrow \mathbb{1}$, that is, a type that only contains values that are $\lambda$-abstractions) returns the domain $\operatorname{dom}(t)$ of the functions in that type and (ii) the infix binary application operator $\circ$, called the result type operator, that given a function type $t$ and a type $s$ subtype of $\operatorname{dom}(t)$ returns the type $t \circ s$ of all values resulting from the application of a function of type $t$ to an argument of type $s$. To explain why lifting these operators to gradual types is challenging let us consider the $\operatorname{dom}($.$) operator (but a similar argument holds for the result type$ operator too).

If we do not have any type connective, then, in general, all function types are of the form $s \rightarrow t$. Therefore defining the domain and result operators is trivial: $\operatorname{dom}(s \rightarrow t)$ is $s$ (the type on the left of the arrow) and if $s^{\prime} \leq s$, then $(s \rightarrow t) \circ s^{\prime}$ is $t$ (the type on the right of the arrow). Adding type connectives makes such definitions more difficult since one must determine what is the domain of, say, the function type $(($ Int $\rightarrow$ Bool $) \wedge \neg$ Int $) \vee(\neg($ Bool $\rightarrow$ Int $) \wedge($ Int $\rightarrow$ Int $))$ or what is the type of the result when a function of this type is applied to some argument. The solution to this problem is given by Frisch et al. [2008] and consists in transforming every function type into an equivalent - with respect to $\simeq$, the equivalence relation induced by $\leq-$ type in disjunctive normal form, which is a type formed by unions of intersections of literals (ie, of atoms or their negation). For such a type it is then easy to define $\operatorname{dom}()$ (eg, the domain of an intersection of arrows is the union of the domains of the arrows; the domain of a union of arrows is the intersection of their domains) and, to a lesser extent, $\circ$. Unfortunately it is not possible to adapt this technique to gradual types: since the transitive closure of $\widetilde{\leq}$ is the trivial full binary relation on GTypes, then it is unsound to consider, as with static types, the equivalence relation induced by $\widetilde{\leq}$ (since it is the full relation too). The solution we propose is to define a different concretization function for gradual types called applicative concretization ${ }^{9}$ that is tailored to the definition of the two operators at issue. The resulting definitions are quite technical and barely intuitive but they have the properties we seek for, since they allow us to define in Section 3 a type-inference system that enjoys the subject-reduction and progress properties. We first give the formal definitions, followed by an intuitive explanation, and then give an a posteriori justification of our definitions by showing that our new concretization function corresponds to transforming gradual types into gradual normal forms (that are the natural gradual extension of the normal forms for static types) by applying a rewriting system that preserves the semantics of $\operatorname{dom}($.$) and \circ$. For space reasons we just hint at this a posteriori justification: all details can be found in the appendix available on-line.

\footnotetext{
${ }^{9}$ Strictly speaking, what we are going to define is not a concretization function, in the sense of abstract interpretation, but rather a function that distills the functional characteristics of a given type.
}

Proc. ACM Program. Lang., Vol. 1, No. ICFP, Article 41. Publication date: September 2017. 
The applicative concretization is defined as follows (where $\mathscr{P}_{f}$ denotes the set of finite subsets):

Definition 4. (Applicative Concretization) The applicative concretization $\gamma_{\mathscr{A}}^{+}$of a gradual type $\tau$ is defined as:

$$
\begin{array}{rlrl}
\gamma_{\mathscr{A}}^{+}: \text {GTypes } & \rightarrow \mathscr{P}_{f}\left(\mathscr{P}_{f}(\text { GTypes })\right) & \gamma_{\mathscr{A}}^{-}: \text {STypes } & \rightarrow \mathscr{P}_{f}\left(\mathscr{P}_{f}\right. \text { (STypes)) } \\
\gamma_{\mathscr{A}}^{+}\left(\tau_{1} \vee \tau_{2}\right) & =\gamma_{\mathscr{A}}^{+}\left(\tau_{1}\right) \cup \gamma_{\mathscr{A}}^{+}\left(\tau_{2}\right) & \gamma_{\mathscr{A}}^{-}\left(t_{1} \vee t_{2}\right) & =\left\{T_{1} \cup T_{2} \mid T_{i} \in \gamma_{\mathscr{A}}^{-}\left(t_{i}\right) \text { for } i=1,2\right\} \\
\gamma_{\mathscr{A}}^{+}\left(\tau_{1} \wedge \tau_{2}\right) & =\left\{T_{1} \cup T_{2} \mid T_{i} \in \gamma_{\mathscr{A}}^{+}\left(\tau_{i}\right) \text { for } i=1,2\right\} & \gamma_{\mathscr{A}}^{-}\left(t_{1} \wedge t_{2}\right) & =\gamma_{\mathscr{A}}^{-}\left(t_{1}\right) \cup \gamma_{\mathscr{A}}^{-}\left(t_{2}\right) \\
\gamma_{\mathscr{A}}^{+}(\sigma \rightarrow \tau) & =\{\{\sigma \rightarrow \tau\}\} & \gamma_{\mathscr{A}}^{-}(s \rightarrow t) & =\{\emptyset\} \\
\gamma_{\mathscr{A}}^{+}(\neg t) & =\gamma_{\mathscr{A}}^{-}(t) & \gamma_{\mathscr{A}}^{-}(\neg t) & =\gamma_{\mathscr{A}}^{+}(t) \\
\gamma_{\mathscr{A}}^{+}(\mathbb{0}) & =\emptyset & \gamma_{\mathscr{A}}^{-}(\mathbb{D}) & =\{\emptyset\} \\
\gamma_{\mathscr{A}}^{+}(\mathbb{1}) & =\{\emptyset\} & \gamma_{\mathscr{A}}^{-}(\mathbb{1}) & =\emptyset \\
\gamma_{\mathscr{A}}^{+}(b) & =\{\emptyset\} & \gamma_{\mathscr{A}}^{-}(b) & =\{\emptyset\} \\
\gamma_{\mathscr{A}}^{+}(?) & =\{\{? \rightarrow ?\}\} &
\end{array}
$$

Notice that that both functions produce sets of (respectively, gradual and static) arrow types.

Next we use the applicative concretization to define the domain and result operators on gradual types:

Definition 5. (Gradual Type Operators) Let $\tau$ and $\sigma$ be two gradual types such that $\tau \widetilde{\mathbb{O}} \rightarrow \mathbb{1}$ and $\sigma \widetilde{\leq} \widetilde{\operatorname{dom}}(\tau)$. The gradual domain of $\tau$, noted $\widetilde{\operatorname{dom}}(\tau)$ and the gradual result type of the application of $\tau$ to $\sigma$, noted $\tau \widetilde{\sigma} \sigma$ are respectively defined as follows:
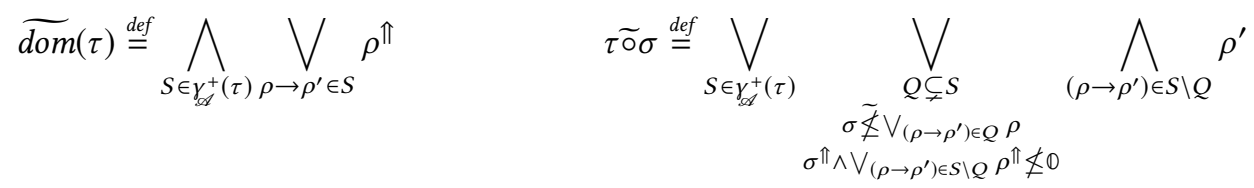

In order to explain and justify the definitions above, let us recall the details of how the operators of domain and result are defined for set-theoretic types [Frisch et al. 2008] and, thus, for our static types. Given a static function type $t$ (ie, a type $t$ such that $t \leq \mathbb{0} \rightarrow \mathbb{1}$ ) its domain $\operatorname{dom}(t)$ is semantically defined in [Frisch et al. 2008] as the greatest type $t^{\prime}$ such that $t \leq t^{\prime} \rightarrow \mathbb{1}$. Such a type is uniquely defined modulo the equivalence relation $\simeq$ and its definition is preserved by this equivalence, that is, two equivalent types have the same domain. Given a static type $t$, there exists a type equivalent to $t$ that is in disjunctive normal form, that is, a type which is a union of uniform intersections ${ }^{10}$ of atoms or their negations. In particular if $t$ is a function type, then all its disjunctive normal forms have the following shape:

$$
t \simeq \bigvee_{f \in F} \bigwedge_{j \in P_{f}} s_{j} \rightarrow t_{j} \wedge \bigwedge_{n \in N_{f}} \neg\left(s_{n} \rightarrow t_{n}\right)
$$

Frisch et al. [2008] prove that the domain of the disjunctive normal form above is equivalent to

$$
\bigwedge_{f \in F} \bigvee_{j \in P_{f}} s_{j}
$$

and since the domain is preserved by $\simeq$, then the type in (2) is also equivalent to $\operatorname{dom}(t)$, the domain of $t$. Since for every type $t$ it is possible effectively to compute a disjunctive normal form equivalent to it, then the domain of a function type can be effectively computed, too. Frisch et al. [2008] proceed similarly for the result type: if $t$ is a functional type with a disjunctive normal form as in

\footnotetext{
${ }^{10}$ Uniform means that the atoms in the intersections are either all arrows or all basic types
} 
(1) and $s$ is a subtype of $d o m(t)$, then the result type $t \circ s$ is defined semantically (as the least type $t^{\prime}$ such that $t \leq s \rightarrow t^{\prime}$ ), it is preserved by equivalence, and it is proved to be equivalent to:

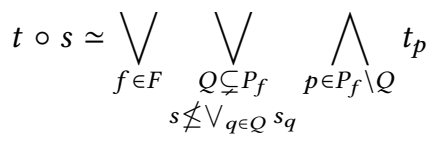

We do not explain the formula above: the details are given by Frisch et al. [2008] (a simpler and more detailed presentation is given by Castagna [2015, sect. 4.4.3]). We just invite the reader to recognize in (2) and (3) the patterns of the formulæ given in Definition 5 and see that what the applicative concretization of Definition 4 does is nothing but to transform a gradual type into a particular disjunctive normal form represented as a finite set (the union) of finite sets (the intersections) of arrow types. This correspondence is not just intuitive but is formalized in the appendix. Here we just outline its main ideas.

First, notice that neither (2), the definition of domain, nor (3), the definition of result, depend on the negated arrows of the normal form: the set $N_{f}$ (of Negative atoms) does not occur in them, just the set $P_{f}$ (of $P$ ositive atoms) is used. Since $\gamma_{\alpha}^{+}$yields a normal form tailored for these operators, then it does not produce any negated arrow (they are erased by the case $\left.\gamma_{\propto}^{-}(s \rightarrow t)=\{\emptyset\}\right)$. This becomes evident when one applies $\gamma_{\mathscr{A}}^{+}$to a static functional type $t$ like in (1): $\gamma_{\mathscr{A}}^{+}(t)=\left\{\left\{s_{j} \rightarrow t_{j} \mid j \in P_{f}\right\} \mid f \in F\right\}$, which represents $\bigvee_{f \in F} \bigwedge_{j \in P_{f}} s_{j} \rightarrow t_{j}$, namely, the positive atoms of the normal form.

Second, for what concerns the definition of domain it is easy to see that this precisely corresponds to the definition given in (2) where $\gamma_{\infty}^{+}$gives the decomposition in disjunctive normal form and where the domain of an arrow $\rho \rightarrow \rho^{\prime}$ is assumed to be the set of all the values in all possible concretizations of $\rho$, that is $\rho^{\Uparrow}$.

Third, there is the interpretation of the unknown type ?. Since we are only interested in possible interpretations of gradual types as functions, we interpret ? in the same way as ? $\rightarrow$ ?, hence the definition of $\gamma_{\mathscr{A}}^{+}(?)$.

Fourth and last, the formula for the result type in Definition 5 nearly corresponds to the formula in (3) where $\gamma_{\mathscr{A}}^{+}$gives the decomposition in disjunctive normal form. There are however two differences, both in the definition of the sets $Q$ on which the inner union ranges over. The first one is straightforward: since we are working with gradual types we replaced the relation $\not$ by its consistent lifting $\widetilde{Z}$. The second is much subtler, it is a consequence of the first difference, and requires to understand how the formula in (3) works. Once again, for a detailed explanation of this formula we invite the reader to consult the one given by Castagna [2015, sect. 4.4.3]; for this work it suffices to say that when we remove from $P_{f}$ the arrows that are in $Q$, then the domain of (the intersection of) the arrows that remain has a non-empty intersection with the type of the argument (ie, these remaining arrows form a set of arrows that may handle the argument). This is so because $Q$ always contains arrows that alone cannot completely handle the argument (ie, the type of the argument is not a subtype of the domain of the arrows in $Q$ : this is condition $s \not \leq \bigvee_{q \in Q} s_{q}$ ). The consequence of replacing the gradual relation $\widetilde{\ddagger}$ for its non-gradual counterpart $\not \leq$ is that now $Q$ contains arrows that alone may not completely handle the argument. We must ensure that we are not removing too many arrows, that is, that the arrows we remove cannot turn out (at run-time) to be exactly those that were supposed to handle the argument. In other terms, we have to ensure that, as for the formula in (3), the domain of the arrows that remain has a non-empty intersection with the type of the argument. This is exactly what the condition $\sigma^{\Uparrow} \wedge \bigvee_{\left(\rho \rightarrow \rho^{\prime}\right) \in S \backslash Q} \rho^{\Uparrow} \not \mathbb{O}$ in Definition 5 does.

As an example, consider the function type $(? \rightarrow$ Bool $) \wedge($ Int $\rightarrow$ Int $)$ applied to the type Int. Intuitively, the result type must be Int: either $(i)$ the domain of the first arrow turns out to be 
incompatible with Int, and thus only the second arrow contributes to the result by returning an Int, or (ii) also the first arrow can handle the argument, but then the result must be of type Bool $\wedge$ Int, that is, the empty set of values, which means that the application cannot return any value and thus it must diverge; so if a result is returned by this application, then it will be of type Int. Since the applicative concretization of the function type is the singleton $\{\{? \rightarrow$ Bool; Int $\rightarrow$ Int $\}\}$, then the only set $S$ to consider when computing the result type of the application is $\{? \rightarrow$ Bool; Int $\rightarrow$ Int $\}$ Moreover, Int $\widetilde{\ddagger}$ ? holds, but Int $\widetilde{\&}$ Int does not. As such, the only two choices for $Q \subsetneq S$ are $Q=\emptyset$ (which yields the summand Bool $\wedge$ Int of the result type) and $Q=\{? \rightarrow$ Bool $\}$ (which yields the summand Int of the result type), hence the result type Int $\vee(B o o l \wedge$ Int) which is the result we expected, as it is equivalent to Int.

To justify the need for the second condition, consider this time the same function type but applied to an argument of type Bool. Intuitively, the only possible result type is Bool, since a function of type Int $\rightarrow$ Int cannot be applied to a Boolean. However, since Bool $\widetilde{\not}$ ? and Bool $\widetilde{\not}$ Int, then without the second condition, there would be three possible choices for $Q \subsetneq S$, namely, $Q=\{? \rightarrow$ Bool $\}$, $Q=\{$ Int $\rightarrow$ Int $\}$, and $Q=\emptyset$. This would give the result type Bool $\vee \operatorname{Int} \vee(B o o l \wedge$ Int $)$, which is equivalent to Bool $\vee$ Int. This return type, although sound, is not correct since the application cannot return a value of type Int. The problem comes from the fact that $Q=\{? \rightarrow \mathrm{Bool}\}$ is not a valid choice, since the function types that remain in $S \backslash Q$, that is just Int $\rightarrow$ Int, cannot handle an argument of type Bool. Hence the need for the second condition, which excludes the previous case and ensures that the functions in $S \backslash Q$ will always be able to handle at least some values of the argument type.

The technical justification of Definitions 4 and 5 is that they allow us to define in the next sections a type system that satisfies the subject reduction and progress properties. We can however also give a less technical and more semantic justification of these definitions by showing that they correspond to transposing to gradual types the definitions given for set-theoretic types. In particular, if we transpose the notion of disjunctive normal forms from set-theoretic types to gradual types we obtain gradual types of the following form:

$$
\begin{aligned}
\bigvee_{i \in I_{f, 1}} \bigwedge_{j \in J_{i}} \sigma_{j} & \rightarrow \tau_{j} \wedge \bigwedge_{j \in J_{n}} \neg\left(s_{j} \rightarrow t_{j}\right) & \vee & \bigvee_{i \in I_{f, 2}} \bigwedge_{j \in J_{i}} \sigma_{j} \rightarrow \tau_{j} \wedge \bigwedge_{j \in J_{n}} \neg\left(s_{j} \rightarrow t_{j}\right) \wedge ? \\
& \vee \bigvee_{i \in I_{b, 1}} \bigwedge_{j \in J_{i}} b_{j} \wedge \bigwedge_{j \in J_{n}} \neg b_{j} & \vee & \bigvee_{i \in I_{b, 2}} \bigwedge_{j \in J_{i}} b_{j} \wedge \bigwedge_{j \in J_{n}} \neg b_{j} \wedge ?
\end{aligned}
$$

It is possible to define a rewriting system that transforms every gradual type into a gradual disjunctive normal form (see its definition in the Appendix). This system is strongly normalizing but it is not confluent. This is expected: as a static type is equivalent to several distinct disjunctive normal forms, so the rewriting system maps a gradual type in distinct gradual disjunctive normal forms. This was not a problem with static types since the (semantically defined) domain and result operators were preserved by type equivalence. We cannot have the same result for gradual types since the equivalence relation induced by consistent subtyping is trivial, but we can easily transpose it since we can show (cf. Appendix)that the rewriting system preserves the gradual domain and result operators of Definition 5, thus providing a more "semantic" justification for them. In other terms, the equivalence relation induced by the rewriting systems is a sound approximation of the semantic relation for gradual types, the one induced by the lifting of subtyping being too coarse to be of any use. 


\section{GRADUALLY-TYPED LANGUAGE}

We next define a language that uses the types of the previous section and give the corresponding typing rules.

\subsection{Language Syntax}

3.1.1 Grammar. The gradually typed language we consider here is a typed lambda calculus tailored to set-theoretic types.

Definition 6. (Gradually Typed Lambda Calculus) The terms constituting the gradually typed lambda calculus are defined by the following grammar:

$$
\begin{aligned}
\text { Terms } & e::=x|c| \lambda^{\mathbb{I}} x . e \mid e \text { e } \mid(e \in t) ? e: e \\
\text { Values } & v::=x|c| \lambda^{\mathbb{I}} x . e \\
\text { Interfaces } & \mathbb{I}::=\left\{\sigma_{i} \rightarrow \tau_{i} \mid i \in I\right\} \quad \text { (I a finite set) }
\end{aligned}
$$

In an ML-like language, union types are always tagged, meaning they can be eliminated by pattern matching on their constructors. However, our set-theoretic type system allows the use of untagged unions (for example Int $\vee$ Bool), which can only be eliminated dynamically. Thus, we need to keep a dynamic representation of types, and allow for dynamic type tests which are noted $(e \in t) ? e_{1}: e_{2}$. Such a test will reduce to expression $e_{1}$ if the result of the evaluation of $e$ has type $t$ or to expression $e_{2}$ otherwise. Moreover, note that only static types are allowed to be dynamically checked against. The reason for this is that checking a value $v$ against a gradual type $\tau$ would intuitively amount to checking that the type of $v$ is a subtype of $\tau$, but then it would be the same as checking $v$ against either $\tau^{\Uparrow}$ or $\tau^{\Downarrow}$ (according to the semantics we want to give to the type case), that is, in both cases a static type (which is why we chose to let the programmer unambiguously specify the static type the expression must be checked against).

The second difference from the usual lambda calculus is the use of explicit interfaces in lambda expressions. An interface is simply a set of arrows, which stands for the set of all the types of the function. For instance, in this syntax the fourth definition of $f$ we gave in the introduction becomes $\lambda^{\{\mathrm{Bool} \rightarrow \text { Int } \wedge \text { ? } \rightarrow \text { Int; Bool } \rightarrow \text { Bool } \wedge \text { ? } \rightarrow \text { Bool }\}}$ constant... Giving explicit function types to abstractions allows us to have more precise types. For example, consider the function types $\tau_{1}=($ Int $\rightarrow$ Int $) \wedge($ Bool $\rightarrow$ Bool $)$ and $\tau_{2}=($ Int $\vee$ Bool $) \rightarrow($ Int $\vee$ Bool $)$. Both types have the same domain (that is, Int $\vee$ Bool), and, in fact, every function of type $\tau_{1}$ is also of type $\tau_{2}$, but not vice-versa. Indeed, $\tau_{1}$ is more precise than $\tau_{2}$ : when applying a function of type $\tau_{1}$ to an argument of type Int, we can deduce statically that the result will be of type Int. Whereas, when applying a function of type $\tau_{2}$ to the same argument, the only information we can deduce is that the result will be of type Int $\vee$ Bool. Thus, the former type conveys more information than the latter, but it cannot be expressed if only function parameters are explicitly typed.

3.1.2 Interfaces. With static types, the type of a function is the intersection of the types in its interface, as it is a value of each of those types. For example, the type of a well-typed function having the interface $\{$ Int $\rightarrow$ Int; Bool $\rightarrow$ Bool $\}$ is the intersection $($ Int $\rightarrow$ Int $) \wedge($ Bool $\rightarrow$ Bool) However, with gradual types this interpretation is less clear-cut since, for instance, there are two possible ways to understand the interface $\{$ Int $\rightarrow$ Int; $\rightarrow$ ? $\}$. One could say that a function with this interface returns a value of type Int when applied to an argument of type Int, and returns something else when applied to an argument that is not of type Int. Or, one could interpret the interface as stating that the type of this function is the intersection (Int $\rightarrow$ Int) $\wedge(? \rightarrow$ ?) which, according to the definition of the result type given in the previous section, means that it returns a result of type Int $\wedge$ ? when given an argument of type Int. 


$$
\begin{aligned}
& \frac{x: \tau \in \Gamma}{\Gamma \vdash x: \tau}\left(T_{x}\right) \quad \frac{\Gamma \vdash e_{1}: \tau_{1} \quad \Gamma \vdash e_{2}: \tau_{2} \quad \tau_{1} \widetilde{\leq} \mathbb{O} \rightarrow \mathbb{1} \quad \tau_{2} \widetilde{\leq} \widetilde{\operatorname{dom}}\left(\tau_{1}\right)}{\Gamma \vdash e_{1} e_{2}: \tau_{1} \widetilde{\circ} \tau_{2}}\left(T_{a p p}\right)
\end{aligned}
$$

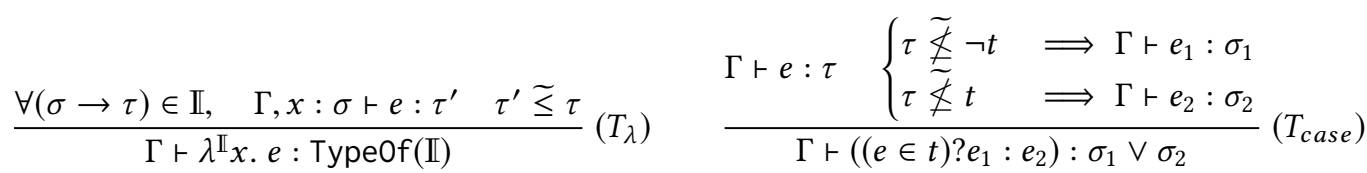

Fig. 1. Typing rules for the gradually typed language

For this work we chose the first approach, since it seems more intuitive and closer to the spirit of gradual types. However, to keep the intuition that the type of a function is the intersection of the types in its interface (and thus ease the formalization), we decided to put a restriction on the interfaces: all the domains (left part) of the arrows of an interface have to be pairwise distinct. Formally, in what follows, we will only consider valid the interfaces $\left\{\sigma_{i} \rightarrow \tau_{i} \mid i \in I\right\}$ such that $\forall(i, j) \in I^{2}, i \neq j \Longrightarrow\left(\sigma_{i} \wedge \sigma_{j}\right)^{\Uparrow} \leq \mathbb{O}$. Moreover, we also impose the condition $\forall i \in I, \sigma_{i}^{\Uparrow} \not \leq \mathbb{O}$, since an arrow of an interface is only meaningful if its domain is not empty. For instance, the interface $\{$ Int $\rightarrow$ Int $; ? \rightarrow$ ? $\}$ is not a valid interface (because Int $\wedge ? \neq \mathbb{0})$, but $\{$ Int $\rightarrow$ Int; $(? \backslash$ Int $) \rightarrow ?\}$ is This definition is not restrictive, as the transformation of an arbitrary interface to a valid interface can be done statically, although this can lead to an exponential blow-up on the size of an interface. For example, the invalid interface $\{\mathrm{Nat} \rightarrow \mathrm{Nat}$; Even $\rightarrow$ Even; $\rightarrow$ ? $\}$ can be converted statically into the (intuitively) equivalent interface

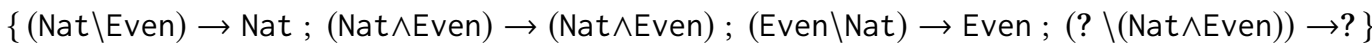

Moreover, interfaces that contain several overlapping gradual types (that is, gradual types that have some concretizations in common) can be rewritten using the same method. For example, the interface $\{(? \rightarrow$ Int; $? \rightarrow$ Bool $\}$ can simply be converted into the interface $\{? \rightarrow$ Int $\vee$ Bool $\}$.

According to this new definition, we will use TypeOf(I) to denote the type associated to a valid interface II, that is, the intersection of its types. Formally, we define the operator TypeOf as follows:

$$
\text { TypeOf(I) }=\bigwedge_{(\sigma \rightarrow \tau) \in \mathbb{I}} \sigma \rightarrow \tau
$$

\subsection{Typing}

Having defined the syntax of the language and the type of lambda expressions, we now use the operators defined in the previous section to provide typing rules for this language. The rules are presented in Figure 1 and assume that we are given a function $B$ that associates to every constant $c$ its static type $B(c)$.

The typing rules $\left(T_{x}\right)$ and $\left(T_{c}\right)$ are the usual rules for typing variables and constants respectively, while the typing rule for lambda expressions $\left(T_{\lambda}\right)$ formalizes the explanations we gave earlier. Notice that, as in any gradual type system, adding a subsumption rule would make the system unsound (since the transitive closure of subtyping is the full relation, then by two consecutive applications of subsumption it would be possible to give any type to every well-typed term). Therefore our systems uses a more "algorithmic presentation" in which the checks of the subtyping relation are distributed over the rules. In particular the rule $\left(T_{\lambda}\right)$, checks that the inferred return type is a subtype of the type specified by the programmer in the interface (ie, $\tau^{\prime} \leq \tau$ which is equivalent to $\left.\tau^{\prime \Downarrow} \leq \tau^{\Uparrow}\right)$. This is used to type functions such as $\lambda^{\{\text {Int } \rightarrow ?\}} x . x$ or, even simpler, $\lambda^{\{\text {Nat } \rightarrow \text { Int }\}} x . x$. 
The typing rule for applications, $\left(T_{a p p}\right)$ is also straightforward, using the definitions of Section 2. Given an application $e_{1} e_{2}$, we just need to ensure that the type $\tau_{1}$ of $e_{1}$ is a function type (ie, a subtype of $\mathbb{D} \rightarrow \mathbb{1}$, which is equivalent to checking $\tau_{1}^{\Downarrow} \leq \mathbb{O} \rightarrow \mathbb{1}$ ), and that the type of $e_{2}$ is a consistent subtype of the domain of $e_{1}$ (equivalently, $\tau_{2}^{\Downarrow} \leq \widetilde{\operatorname{dom}}\left(\tau_{1}\right)$ since the domain always is a static type). The return type of the application is then defined using the result type operator $\widetilde{0}$.

To explain the rule $\left(T_{\text {case }}\right)$, let us first remind the definition of its static counterpart, as defined by Frisch et al. [2008].

$$
\frac{\Gamma \vdash e: t^{\prime} \quad\left\{\begin{array}{l}
t^{\prime} \not \leq \neg t \quad \Longrightarrow \Gamma \vdash e_{1}: s \\
t^{\prime} \not \leq t
\end{array} \Gamma \Gamma \vdash e_{2}: s\right.}{\Gamma \vdash\left((e \in t) ? e_{1}: e_{2}\right): s}
$$

The intuition behind this rule is that if we can statically prove that a branch will not be evaluated, then it does not need to be typed. ${ }^{11}$ Given a type-case $(e \in t) ? e_{1}: e_{2}$, we know that the set of values that $e$ can reduce to is given by the type $t^{\prime}$ of $e$. Therefore, saying that the first branch cannot be evaluated amounts to saying that $t^{\prime} \wedge t \simeq \mathbb{0}$, which is set-theoretically equivalent to $t^{\prime} \leq \neg t$. Thus, the branch $e_{1}$ should be evaluated only if $t^{\prime} \not \leq \neg t$, hence the condition. Naturally, the same reasoning can be done with expression $e_{2}$, providing the second case of this rule.

To deduce the gradual equivalent of this rule, we use the definition of predicate lifting to lift the operator $\not$, which yields the following definition: $\tau \mathbb{\not} \sigma \Longleftrightarrow \exists(t, s) \in \gamma(\tau) \times \gamma(\sigma), t \not \leq s$. As for subtyping, this definition can be simplified using the extrema of concretizations: $\tau \widetilde{\not} \sigma \Longleftrightarrow$ $\tau \Uparrow \not \leq \sigma \Downarrow$. Notice that this definition-ie, the lifting of $\not{\leq}-i$ is not equivalent to the negation of $\widetilde{\leq}$-the lifting of $\leq-$, whose definition is $\neg(\tau \widetilde{\leq} \sigma) \Longleftrightarrow \forall(t, s) \in \gamma(\tau) \times \gamma(\sigma), t \not \leq s \Longleftrightarrow \tau^{\Downarrow} \not \leq \sigma^{\Uparrow}$. The condition $t^{\prime} \not \leq \neg t$ then lifts to the condition $\tau \widetilde{\&} \neg t$ presented in rule $\left(T_{\text {case }}\right)$ which is equivalent to $\tau^{\Uparrow} \not \leq \neg t$ (since $\left.\neg t^{\Downarrow}=\neg t\right)$. Likewise for $t^{\prime} \not \leq t$ and $\tau \not{\not} t$

Rationale: The language we defined in this section is an abstraction of the language the programmer is supposed to program with. Notice that we did not define the semantics of this language and a fortiori we did not prove any soundness or safety property of the type system defined above. The semantics of this language will be given by translating its terms into the "cast language" we define in the next section. The translation is defined in Section 5 where we also prove (cf. Theorem 3 ) that every well-typed term of this language is translated into a well-typed term of the cast language. The soundness of the cast language's type systems (cf. Theorem 2) implies safety (as expressed in Corollary 1) of the type system of Figure 1.

\section{CAST LANGUAGE}

In so-called "sound" gradual typing the compiler must insert dynamic checks into gradually-typed programs to ensure that they do not get stuck at execution. In the previous section we defined our gradually-typed language. In this section we present the target language that includes casts, and give its static and dynamic semantics.

\subsection{Syntax}

The target language we consider is closely related to the gradually-typed lambda calculus that was defined in the previous section.

\footnotetext{
${ }^{11}$ Actually, it must not be typed, otherwise every type-case expression would always be typed by the union of the types of its two cases and the only typeable overloaded functions would be the coherent ones, as in Forsythe [Reynolds 1996]. For instance, it would not be possible to deduce that $\lambda x .(x \in \operatorname{Int})$ ? $(-x)$ :not $(x)$ has type (Int $\rightarrow$ Int $) \wedge($ Bool $\rightarrow$ Bool): see $\S 3.3$ in [Frisch et al. 2008] for a detailed explanation.
} 
Definition 7. (Cast Language) The terms constituting the cast language are defined by the following grammar:

$$
\begin{array}{cl}
\text { Terms }^{\langle\rangle} & e::=x|c| \lambda_{\langle\tau\rangle}^{\mathbb{I}} \text { x. e } \mid \text { e e }|(e \in t) ? e: e|\langle\tau\rangle e \\
\text { Values }^{\backslash\rangle} & v::=c \mid \lambda_{\langle\tau\rangle}^{\mathbb{I}} x . e \\
\text { Interfaces } & \mathbb{I}::=\left\{\sigma_{i} \rightarrow \tau_{i} \mid i \in I\right\} \\
\text { Errors } & \mathscr{E}::=\text { CastError }
\end{array}
$$

As before, every interface $\mathbb{I}=\left\{\sigma_{i} \rightarrow \tau_{i} \mid i \in I\right\}$ must satisfy the conditions $\forall(i, j) \in I^{2},\left(\sigma_{i} \wedge \sigma_{j}\right)^{\Uparrow} \leq$

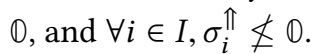

The most important addition of this language to the gradually-typed language of the previous section is the presence of the cast expression $\langle\tau\rangle e$ which verifies whether the value resulting from the evaluation of the expression $e$ has type $\tau$ and returns the value itself or CastError, accordingly.

The other difference is that lambda-abstractions now include cast annotations. The notation $\lambda_{\langle\tau\rangle}^{\mathrm{II}} x . e$ denotes the cast of the function $\lambda^{\mathrm{I}} x . e$ to the type $\tau$. The idea behind this notation is that a lambda expression "stores" a cast in order to evaluate it, only when the function is applied. This lazy evaluation of casts allows us to avoid unnecessary but costly $\eta$-expansions which, as pointed out by Takikawa et al. [2016], hinder the practical interest of sound gradual typing. Moreover, to ease the formalization, we do not include uncast lambda-abstractions of the form $\lambda^{\mathbb{I}} x$. $e$ in the grammar This has two implications. First, this obviously comes at a performance cost, since we may add unnecessary casts to certain functions (most notably, fully statically-typed functions). Second, as a consequence, the compilation procedure will always add casts, even in fully statically-typed programs. However, these casts should never result in a cast error. To ease the notation, we may write $\lambda^{\mathbb{I}} x . e$ as syntactic sugar for an abstraction that stores an identity cast, that is, $\lambda_{\langle\text {TypeOf(II) }\rangle}^{\mathbb{I}} x . e$.

Rationale: Our main objective in this section is to prove the soundness of the cast language, which will allow us to prove that the execution of the compilation of every well-typed term of the gradually-typed language is sound. To achieve this, we start by defining a set of typing rules for the cast language, before giving its operational semantics. We must proceed in this order because the presence of casts and type-cases makes the operational semantics depend on type inference.

\subsection{Typing}

The intuition of the type system in the previous section was that a term is well typed if there exist some concretizations of the gradual types occurring in it for which the execution would succeed. A well-typed term, then, will be compiled into a term of the cast language above by inserting in it all the dynamic checks necessary to ensure the progress property, that is, that its execution will either diverge,$^{12}$ or converge to a value, or raise a cast error. To prove this property of the compilation of a well-typed term, we define a type system for the cast language satisfying the above progress property $-i e$, in which every well-typed converging term yields either a value or a cast error- and then prove that well-typed terms of the gradual language are compiled into well-typed terms of the cast language.

The key case for the type system of the cast language is, as expected, the typing of applications. Consider, for example, an application $e_{1} e_{2}$, where $e_{1}$ is of type ? $\rightarrow$ ? and $e_{2}$ is of type Int. This application is well-typed, since $e_{1}$ is known to be a function that can be applied to any argument, of any type: that fact that $e_{1}$ is well typed with type $? \rightarrow$ ? ensures that $e_{1}$ contains all the casts necessary to guarantee that its argument (of type ?) will not be misused. Consider now the same

${ }^{12}$ An example of well-typed term that diverges is $\omega \omega$ where $\omega \stackrel{\text { def }}{=} \lambda^{\{(? \rightarrow ?) \wedge ? \rightarrow ?\}} . x x$. 
application but where $e_{1}$ is of type ?. This time, this application must not be considered well-typed, since $e_{1}$ is not necessarily a function: at run-time its gradual type may turn out to be, say, Int (eg, if $e_{1}$ reduces to 42) and the application would thus reduce to a stuck term. Correcting this application would require adding a cast: for example, $\left(\langle ? \rightarrow ?\rangle e_{1}\right) e_{2}$, which is well-typed since it is an instance of the previous case (the function $\left(\langle ? \rightarrow ?\rangle e_{1}\right)$ is well typed with type $? \rightarrow$ ?, provided that $e_{1}$ is well typed).

In the absence of type connectives the typing of an application is easily solved: whenever the type $e_{1}$ is not an arrow, the application is not well typed (eg, see the typing of the cast languages in [Siek and Taha 2006; Wadler and Findler 2009]). Type connectives make this issue more difficult. Consider again the application $e_{1} e_{2}$ and suppose that the type of $e_{1}$ is $($ Int $\rightarrow$ Int $) \wedge ?$ ? In the gradually-typed language of Section 3 this application is well-typed since the domain of this function is $\mathbb{1}$ : this function can be applied to any argument. However, in the cast language the situation is quite different, since whether this application is to be considered well-typed or not depends on the type of $e_{2}$. If for instance $e_{2}$ is of type Int, then we know that this application cannot fail since the type (Int $\rightarrow$ Int) $\wedge$ ? guarantees that $e_{2}$ is a function that can (at least) be applied to integer arguments. If instead $e_{2}$ is, say, of type Bool, then this application may fail, notably in the case that ? does not turn out to be a subtype of Bool $\rightarrow \mathbb{1}$. Therefore this application is not a well-typed term of the cast language, even though it could be transformed into one at compile time by a suitable cast of the function $\left(e g,\left(\langle\mathrm{Bool} \rightarrow \mathbb{1}\rangle e_{1}\right) e_{2}\right)$. This example shows that, even if the domain of a function of type (Int $\rightarrow$ Int $) \wedge$ ? is $\mathbb{1}$, we are guaranteed that the application of such a function will not fail only if the argument is of type Int. We say that Int is the safe domain of the functions of type (Int $\rightarrow$ Int $) \wedge$ ?, that is, what we call safe domain is the type of all the arguments for which the application of these functions cannot get stuck (ie, all the arguments for which the functions need not to be cast). In particular, while the domain of ? is $\mathbb{1}$, the safe domain of ? is $\mathbb{0}$, since it is not possible to guarantee that the application of a "function" of type ? will not fail, whatever argument we use. Likewise, the domain and safe domain of $? \rightarrow$ ? are both $\mathbb{1}$ : a function of type $? \rightarrow$ ? contains in its body all the casts necessary to ensure that the execution will not get stuck, whatever argument the function is applied to. To define the safe domain operator, we replicate the method used in Section 2 to define the domain operator, and start by giving a new concretization function.

Definition 8. (Safe Applicative Concretization) For every gradual type $\tau$, we define the safe applicative concretization $\gamma_{\mathscr{S}}^{+}$of $\tau$ as follows:

$$
\begin{aligned}
\gamma_{\mathscr{S}}^{+}: \text {GTypes } & \rightarrow \mathscr{P}_{f}\left(\mathscr{P}_{f} \text { (GTypes) }\right) \\
\gamma_{\mathscr{S}}^{+}\left(\tau_{1} \vee \tau_{2}\right) & =\gamma_{\mathscr{S}}^{+}\left(\tau_{1}\right) \cup \gamma_{\mathscr{S}}^{+}\left(\tau_{2}\right) \\
\gamma_{\mathscr{S}}^{+}\left(\tau_{1} \wedge \tau_{2}\right) & =\left\{T_{1} \cup T_{2} \mid T_{i} \in \gamma_{\mathscr{S}}^{+}\left(\tau_{i}\right) \text { for } i=1,2\right\} \\
\gamma_{\mathscr{S}}^{+}(\sigma \rightarrow \tau) & =\{\{\sigma \rightarrow \tau\}\} \\
\gamma_{\mathscr{S}}^{+}(\neg t) & =\gamma_{\mathscr{S}}^{-}(t) \\
\gamma_{\mathscr{S}}^{+}(\mathbb{D}) & =\emptyset \\
\gamma_{\mathscr{S}}^{+}(\mathbb{1}) & =\{\emptyset\} \\
\gamma_{\mathscr{S}}^{+}(b) & =\{\emptyset\} \\
\gamma_{\mathscr{S}}^{+}(?) & =\{\{\mathbb{0} \rightarrow ?\}\}
\end{aligned}
$$

$$
\begin{aligned}
\gamma_{\mathscr{S}}^{-}: \text {STypes } & \rightarrow \mathscr{P}_{f}\left(\mathscr{P}_{f}(\text { STypes })\right) \\
\gamma_{\mathscr{S}}^{-}\left(t_{1} \vee t_{2}\right) & =\left\{T_{1} \cup T_{2} \mid T_{i} \in \gamma_{\mathscr{S}}^{-}\left(t_{i}\right) \text { for } i=1,2\right\} \\
\gamma_{\mathscr{S}}^{-}\left(t_{1} \wedge t_{2}\right) & =\gamma_{\mathscr{S}}^{-}\left(t_{1}\right) \cup \gamma_{\mathscr{S}}^{-}\left(t_{2}\right) \\
\gamma_{\mathscr{S}}^{-}(s \rightarrow t) & =\{\emptyset\} \\
\gamma_{\mathscr{S}}^{-}(\neg t) & =\gamma_{\mathscr{S}}^{+}(t) \\
\gamma_{\mathscr{S}}^{-}(\mathbb{O}) & =\{\emptyset\} \\
\gamma_{\mathscr{S}}^{-}(\mathbb{1}) & =\emptyset \\
\gamma_{\mathscr{S}}^{-}(b) & =\{\emptyset\}
\end{aligned}
$$

The only difference between $\gamma_{\mathscr{A}}^{+}$and $\gamma_{\mathscr{S}}^{+}$is the concretization of ? which the latter concretizes using only its safe domain. Next, we use this definition to define the safe domain operator, similarly to the domain operator. 
Definition 9. (Safe Gradual Domain) For every gradual type $\tau$ verifying $\tau \widetilde{\leq} \mathbb{O} \rightarrow \mathbb{1}$, we define the safe gradual domain of $\tau$, noted $\widetilde{d o m}_{\mathscr{\rho}}(\tau)$, as follows:

$$
\widetilde{\operatorname{dom}}_{\rho^{\prime}}(\tau)=\left\{\begin{array}{cl}
\bigwedge_{S \in \gamma_{S}^{+}(\tau)} \bigvee_{\sigma \rightarrow \rho \in S} \sigma^{\Uparrow} & \text { if } \tau^{\Uparrow} \leq \mathbb{0} \rightarrow \mathbb{1} \\
0 & \text { otherwise }
\end{array}\right.
$$

This definition is the same as the definition of the domain given in Section 2 except an important difference: if $\tau \Uparrow \not \leq \mathbb{O} \rightarrow \mathbb{1}$, then the safe domain of $\tau$ is defined as $\mathbb{O}$. This formalizes the intuition that, if $\tau$ is not always a function type (ie, there exists a concretization of $\tau$ that is not a function type), then its safe domain is empty.

The definition of safe domain allows us to type applications in the cast language, but there still is a class of terms of the cast language that poses a problem: the lambda-abstractions. The problem comes from the use of interfaces and can be illustrated by considering the rule $\left(T_{\lambda}\right)$ in Figure 1 which shows that once we have inferred the type of the body of the function, we still have to check that this type is a consistent subtype of the type the programmer wrote in the interface, insofar as the latter is the one used to type the function. The situation is similar for the abstractions in the cast language, but in this case consistent subtyping is not strict enough to ensure progress. Consider for instance a lambda-abstraction of the cast language of the form $\lambda^{\{\text {Int } \rightarrow \text { Int } \rightarrow \text { Int }\}} x . e$ (for the sake of simplicity we omitted the cast in the lambda abstraction: consider it to be the identity cast). If this lambda expression is well-typed, then so is the double application $\left(\left(\lambda^{\{\operatorname{Int} \rightarrow \operatorname{Int} \rightarrow \operatorname{Int}\}} x . e\right) 3\right) 5$. However requiring that the type of the body $e$ is a consistent subtype of Int $\rightarrow$ Int does not suffice to ensure progress. Take for $e$ the expression $\langle$ ? $\rangle \mathrm{x}$ : this expression has type ? and ? $\widetilde{\leq}$ Int $\rightarrow$ Int, nevertheless the application above would return (3)5, a stuck expression. Therefore, to compare the type of the body with the one recorded in the interface we need a relation $\sqsubseteq$ that is strictly finer than consistent subtyping. The problem can be reframed into a more general one, namely, that consistent subtyping does not satisfy the substitution property: replacing in a given context a term of some gradual type by a term of a consistent subtype does not preserve progress. Hence the need for a stronger relation $\sqsubseteq$ on types which enjoys the substitution property and is preserved during the evaluation of a term: for instance, in the example $\lambda^{\{\text {Int } \rightarrow \text { Int } \rightarrow \text { Int }\}} x$. $e$ above we want that whatever value $e$ reduces to, it will preserve progress in a context where an expression of type Int $\rightarrow$ Int is expected. In particular, this relation has to be transitive (which is not the case of consistent subtyping), and must retain enough information about function types so that reducing under an application still produces a well-typed term. Consider a term $e$ of type $\tau$ that reduces into a term $e^{\prime}$ of type $\tau^{\prime}$. First of all, we want to make sure that we did not lose information when reducing $e$, or that $\tau^{\prime}$ is more precise than $\tau$. This amounts to saying that every concretization of $\tau^{\prime}$ is also a concretization of $\tau$, which can be written as $\tau^{\prime \Uparrow} \leq \tau^{\Uparrow}$. Note that this relation is transitive, and it implies subtyping. Secondly, if $\tau$ is always a function type $(i e, \tau \Uparrow \leq \mathbb{O} \rightarrow \mathbb{1})$, then although the previous condition implies that $\tau^{\prime}$ is also a function type, it does not say anything about the applicative concretizations of $\tau$ and $\tau^{\prime}$. Thus we do not know anything about the relation between the domains and results of $\tau$ and $\tau^{\prime}$. We want to make sure that the subtyping relation between $\tau$ and $\tau^{\prime}$ also translates to their applicative concretizations. Formally, this can be written as the condition:

$$
\gamma_{\mathscr{S}}^{+}\left(\tau^{\prime}\right) \subset \gamma_{\mathscr{S}}^{+}(\tau)
$$

Using the intuition that the applicative concretization is representing a normal form, this amounts to saying that if $\tau$ is a union of intersections, then $\tau^{\prime}$ is a union of fewer intersections than $\tau$. The 


$$
\begin{aligned}
& \frac{x: \tau \in \Gamma}{\Gamma \vdash x: \tau}\left(T_{x}^{\langle\rangle}\right) \quad \frac{\text { Type0f(I) } \widetilde{\leq} \tau}{\Gamma(\sigma \rightarrow \rho) \in \mathbb{I}, \quad \Gamma, x: \sigma \vdash e: \rho^{\prime} \quad \rho^{\prime} \sqsubseteq \rho}\left(T_{\lambda}^{\langle\rangle}\right) \\
& \frac{\Gamma \vdash e_{1}: \tau_{1} \quad \Gamma \vdash e_{2}: \tau_{2} \quad \tau_{1}^{\Uparrow} \leq \mathbb{0} \rightarrow \mathbb{1} \quad \tau_{2}^{\Uparrow} \leq \widetilde{\operatorname{dom}}_{\mathscr{f}}\left(\tau_{1}\right)}{\Gamma \vdash e_{1} e_{2}: \tau_{1} \widetilde{o} \tau_{2}}\left(T_{c}^{\langle\rangle}\right) \quad
\end{aligned}
$$

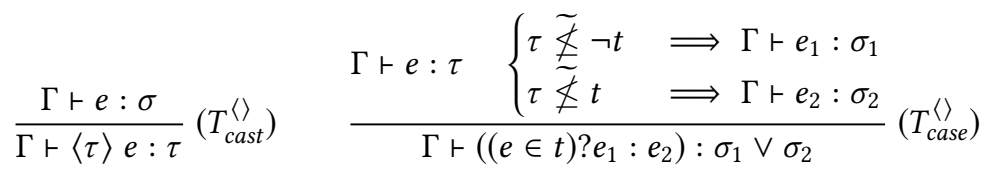

Fig. 2. Typing rules for the cast language

same reasoning can be done with the result types, which gives the same condition but with $\gamma_{\alpha}^{+}$. Hence the definition of the applicative subtyping.

Definition 10. (Applicative Subtyping) For every gradual types $\sigma$ and $\tau$ such that $\sigma^{\Uparrow} \leq \mathbb{0} \rightarrow \mathbb{1}$ and $\tau^{\Uparrow} \leq \mathbb{O} \rightarrow \mathbb{1}$, we define the applicative subtyping relation as follows:

$$
\sigma \leq \tau \Longleftrightarrow\left\{\begin{array}{l}
\gamma_{\mathscr{S}}^{+}(\sigma) \subset \gamma_{\mathscr{S}}^{+}(\tau) \\
\gamma_{\mathscr{A}}^{+}(\sigma) \subset \gamma_{\mathscr{A}}^{+}(\tau)
\end{array}\right.
$$

Note that the subset relation used in this definition uses the syntactic equality over types. That is, $\{\{$ Int $\rightarrow$ Int $\}\} \subset\{\{$ Int $\rightarrow$ Int $\} ;\{$ Bool $\rightarrow$ Bool $\}\}$ but $\{\{$ Int $\rightarrow$ Int $\}\} \not \subset\{\{$ Nat $\rightarrow$ Int $\}\}$ even if Int $\rightarrow$ Int $\leq$ Nat $\rightarrow$ Int. We are only interested in finding a possible subtyping relation that has the required properties (substitution property and preservation during evaluation), not in finding the most general one. As such, we chose to only consider the syntactic equality between types since it makes the relation general enough and easier to use than a subtyping-based definition.

We can then define the compatible subtyping, which possesses all the properties we are looking for, as it will be formalized in Theorem 1 .

Definition 11. (Compatible Subtyping) For every gradual types $\sigma$ and $\tau$, we define the compatible subtyping $\sqsubseteq$ as follows:

$$
\sigma \sqsubseteq \tau \Longleftrightarrow\left\{\begin{array}{l}
\sigma^{\Uparrow} \leq \tau^{\Uparrow} \\
\tau^{\Uparrow} \leq \mathbb{0} \rightarrow \mathbb{1} \Longrightarrow \sigma \leq \tau
\end{array}\right.
$$

The typing rules for the cast language are presented in Figure 2, and are directly derived from the rules of the gradually-typed language presented in Section 3. The differences are the addition of a rule $\left(T_{\text {cast }}^{\langle\rangle}\right)$for the typing of cast expressions and the modifications of the rules $\left(T_{a p p}^{\langle\rangle}\right)$and $\left(T_{\lambda}^{\langle\rangle}\right)$ in the way we just outlined. In particular, the example we discussed above, $\lambda^{\{\text {Int } \rightarrow \operatorname{Int} \rightarrow \operatorname{Int}\}} x .\langle ?\rangle x$, is not well typed because ? $\nsubseteq$ Int $\rightarrow$ Int.

The rule $\left(T_{\text {cast }}^{\langle\rangle}\right)$is straightforward and states that the expression $\langle\tau\rangle e$ has type $\tau$, independently of the type of $e$. The reason for that is that if $e$ converges, then either $e$ will reduce to a value of type $\tau$-and so the whole expression will- or a cast error will be raised: in either cases the expression will not be stuck.

The rule $\left(T_{a p p}^{\langle\rangle}\right)$is modified to ensure that the type on the left-hand side of an application is always a function type, that is, all of its concretizations are a subtype of $\mathbb{O} \rightarrow \mathbb{1}$. This translates 
to the condition $\tau_{1}^{\Uparrow} \leq \mathbb{0} \rightarrow \mathbb{1}$. Moreover, we also want to ensure that the type of the argument is always accepted by the function, which amounts to saying that all its concretizations are in the safe domain of the function. Hence the condition $\tau_{2}^{\Uparrow} \leq \widetilde{\operatorname{dom}_{\rho}}\left(\tau_{1}\right)$.

Finally, rule $\left(T_{\lambda}^{\langle\rangle}\right)$returns the type of the cast embedded in the lambda abstraction and uses the stricter compatible subtyping relation $\sqsubseteq$ to check that the body of the abstraction is compatible with the one written in the interface.

As a result, this type system enjoys a substitution property with respect to the compatible subtyping, which is formalized in the following theorem.

Theorem 1. (Substitution) For every terms of the cast language $e, e^{\prime} \in$ Terms $^{\langle\rangle}$and every typing context $\Gamma$, if $\Gamma, x: \sigma \vdash e: \tau$ and $\Gamma \vdash e^{\prime}: \sigma^{\prime}$ where $\sigma^{\prime} \sqsubseteq \sigma$, then $\Gamma \vdash e\left[x:=e^{\prime}\right]: \tau^{\prime}$ and $\tau^{\prime} \sqsubseteq \tau$.

Finally, notice that the inference rules of Figure 2 are deterministic: the system infers a unique type for every well-typed expression. This property is important since type inference is used in the next subsection to define the operational semantics which, thanks to this property, will be deterministic, too.

\subsection{Operational Semantics}

The small-step reduction rules for the cast language are presented in Figure 3 and are divided in four categories: cast, type-case, application, and context.

The definition of the reduction contexts at the bottom of the figure corresponds to a leftmost outermost weak reduction strategy which is applied by the two context reduction rules, $\left(R_{E}\right)$ and $\left(R_{E-\text { fail }}\right)$, which respectively propagate the computation and raise the cast errors at top-level.

The reduction rules for type casts applied to constants, $\left(R_{\text {cast }-c}\right)$ and $\left(R_{\text {cast }-c-f a i l}\right)$, are straightforward: if the type of the constant is a subtype of the target type, then the cast succeeds and the constant is returned; otherwise, the cast fails and the expression reduces to a cast error. The cast rules for lambda-abstractions $\left(R_{\text {cast }-\lambda}\right)$ and $\left(R_{\text {cast }-\lambda-\text { fail }}\right)$ look similar. However, there is an important difference, that is, that casts for lambda-abstractions are evaluated lazily at the moment of their application. So instead of performing an $\eta$-expansion, as it is customary in cast languages for gradual typing, the rule $\left(R_{\text {cast }-\lambda}\right)$ "stores" the cast in the lambda-expression to evaluate it later. Note that if a lambda expression is preceded by multiple casts, then the rule $\left(R_{\text {cast }-\lambda}\right)$ erases all of them, except for the last one. Indeed, storing all the successive casts of a function would introduce a chain of casts when computing the result of an application, where the only relevant cast would be the outermost one (as it represents the type that is expected by the rest of the program). Therefore, removing these casts preserves the soundness of the evaluation while reducing the number of possible cast errors. Notice that if we can already statically determine that a given cast will always fail $-i e$, because the cast type is not a consistent subtype of the interface-, then the $\left(R_{\text {cast }-\lambda-\text { fail }}\right)$ rule raises outright the cast error.

The rule $\left(R_{\text {case }-L}\right)$ is slightly less intuitive. If $v$ is a value, then the semantics of the expression $\left((v \in t) ? e_{1}: e_{2}\right)$ in a language without gradual types consists in checking whether the type of $v$ is a subtype of $t$ (since, by subsumption, this implies that the value has type $t$ ) and executing $e_{1}$ if so, and $e_{2}$ otherwise. As we explained in Footnote 2, this test can be efficiently implemented by using the type information available at compile time. In the presence of gradual types, checking the subtyping relation between the type $\tau$ of $v$ and $t$ (ie, $\tau^{\Downarrow} \leq t$ ), although sound, is not the best choice for selecting $e_{1}$, since it might yield the insertion of casts that will always fail. Instead we have to select $e_{1}$ only if the type of $v$ is always a subtype of $\tau$, that is, whatever concretization its type will turn out to be $\left(i e, \tau^{\Uparrow} \leq t\right)$. This is done by the rule $\left(R_{\text {case }-L}\right)$, while rule $\left(R_{\text {case }-R}\right)$ corresponds to the 


\section{Cast}
$\frac{B(c) \widetilde{\leq} \tau}{\langle\tau\rangle c \mapsto c}\left(R_{\text {cast }-c}\right)$
$\frac{\neg(B(c) \widetilde{\leq} \tau)}{\langle\tau\rangle c \mapsto \text { CastError }}\left(R_{\text {cast }-c-f a i l}\right)$
$\frac{\text { TypeOf(I) } \widetilde{\leq} \tau}{\langle\tau\rangle \lambda_{\left\langle\tau^{\prime}\right\rangle}^{\mathbb{I}} x . e \mapsto \lambda_{\langle\tau\rangle}^{\mathbb{I}} x . e}\left(R_{\text {cast }-\lambda}\right)$
$\frac{\neg(\text { TypeOf }(\mathbb{I}) \widetilde{\leq} \tau)}{\langle\tau\rangle \lambda_{\left\langle\tau^{\prime}\right\rangle}^{\mathrm{I}} x . e \mapsto \text { CastError }}\left(R_{\text {cast }-\lambda-\text { fail }}\right)$

\section{Type-case}

$$
\frac{\emptyset \vdash v: \tau \quad \tau^{\Uparrow} \leq t}{\left((v \in t) ? e_{1}: e_{2}\right) \mapsto e_{1}}\left(R_{\text {case }-L}\right) \quad \frac{\emptyset \vdash v: \tau \quad \tau^{\Uparrow} \not \leq t}{\left((v \in t) ? e_{1}: e_{2}\right) \mapsto e_{2}}\left(R_{\text {case }-R}\right)
$$

\section{Application}

$$
\begin{array}{lc}
\frac{\exists\left(\sigma_{i} \rightarrow \tau_{i}\right) \in \mathbb{I} \quad B(c) \widetilde{\leq} \sigma_{i}}{\left(\lambda_{\langle\tau\rangle}^{\mathbb{I}} x . e\right) c \mapsto\langle\tau \widetilde{\circ} B(c)\rangle e\left[x:=\left\langle\sigma_{i}\right\rangle c\right]}\left(R_{a p p-c}\right) & \frac{\nexists\left(\sigma_{i} \rightarrow \tau_{i}\right) \in \mathbb{I} \quad B(c) \leq \sigma_{i}^{\Uparrow}}{\left(\lambda_{\langle\tau\rangle}^{\mathbb{I}} x . e\right) c \mapsto \text { CastError }}\left(R_{a p p-c-f a i l}\right) \\
\frac{\nexists\left(\sigma_{i} \rightarrow \tau_{i}\right) \in \mathbb{I} \quad B(c) \leq \sigma_{i}^{\Uparrow}}{\left(\lambda_{\langle\tau\rangle}^{\mathbb{I}} x . e\right) c \mapsto \text { CastError }}\left(R_{a p p-c-f a i l}\right) & \frac{\nexists\left(\sigma_{i} \rightarrow \tau_{i}\right) \in \mathbb{I}}{\left(\lambda_{\langle\tau\rangle}^{\mathbb{I}} x . e\right)\left(\lambda_{\left\langle\tau^{\prime}\right\rangle}^{\mathbb{I}^{\prime}} y . e^{\prime}\right) \mapsto \text { CastError }} \quad \text { TypeOf }\left(\mathbb{I}^{\prime}\right)^{\Downarrow} \leq \sigma_{i}^{\Uparrow}
\end{array}
$$

\section{Context}

$$
\begin{gathered}
\frac{e \mapsto e^{\prime}}{E[e] \mapsto E\left[e^{\prime}\right]}\left(R_{E}\right) \quad \frac{e \mapsto \text { CastError }}{E[e] \mapsto \text { CastError }}\left(R_{E-f a i l}\right) \\
E::=\square|E e| v E|(E \in t) ? e: e|\langle\tau\rangle E
\end{gathered}
$$

Fig. 3. Small-step reduction semantics for the cast language

other case. To understand the need of this stricter check consider the following expression

$$
\left(\left(f=\lambda^{\{? \rightarrow ?\}} x .(1+\langle\text { Int }\rangle x) \in(\text { Bool } \rightarrow \text { Bool })\right) ?(f \text { true }):\right. \text { false }
$$

that checks whether $f$ is of type Bool $\rightarrow$ Bool, applies it to true if this holds, and return false otherwise. In this term $f$ is bound to a dynamically-typed version of the successor function, since it is given type ? $\rightarrow$ ?. This type is a consistent subtype of Bool $\rightarrow$ Bool. However, since $(? \rightarrow ?)^{\Uparrow}=\mathbb{O} \rightarrow \mathbb{1}$ is not a subtype of Bool $\rightarrow$ Bool, then the reduction rule $\left(R_{\text {case }-R}\right)$ applies and the second branch is selected, which is arguably the correct choice. Selecting the first branch, as the simpler subtyping check would do, would yield to a term that always fails since it would try to cast the argument of $f$, that is true, to Int. ${ }^{13}$

The rules $\left(R_{a p p-*}\right)$ for application are the most difficult ones. First of all, remember that we evaluate function casts in a lazy way and, in particular, when the functions are applied. So the application rules perform two operations at once: the substitution corresponding to the beta reduction, and the

\footnotetext{
${ }^{13}$ Notice that for this example we used an extended syntax of type case in which the tested term is also bound to a variable. As explained in Section E in the Appendix of [Castagna et al. 2014] this extended syntax can be encoded in our syntax and provides a finer-grained typing for the type-case expression, similar to the occurrence typing of Tobin-Hochstadt and Felleisen [2008].
} 
evaluation of the cast embedded in the function. The latter consists in $\eta$-expanding the function to apply the appropriate casts to its argument and to its result, and we perform it directly on the reductum of the beta-reduction. More precisely, when evaluating the expression $\left(\lambda_{\langle\tau\rangle}^{\mathbb{I}} x . e\right) v$ the lambda-expression expects an argument whose type is a subtype of its domain, not of $\widetilde{\operatorname{dom}}(\tau)$ (which is the domain of the cast function). As such, $x$ cannot simply be substituted by $v$ in the expression $e$ : an additional check must be performed on $v$. Hence, we look in the interface for an arrow $\sigma_{i} \rightarrow \tau_{i}$ whose input type $\sigma_{i}$ is compatible with the type of $v$ and we substitute for $x$ the expression $\left\langle\sigma_{i}\right\rangle v$ (or its reductum). Two observations: first, this target type $\sigma_{i}$, if it exists, is unique thanks to the property that interfaces have pairwise disjoint input types. Second, if the argument is a lambda-abstraction, then the cast it contains is erased by the cast to the target type, and thus the former does not dictate how the argument can be used in the body of the function. Therefore, the choice of the target type should not depend on the cast contained by the argument (since it is erased) but on the interface contained in the argument, which explains why we had to split the application rule in two subcases, one for constants (which uses the type of the argument to select the target type) and the other for abstractions (which uses the interfaces of the argument to select the target type). Notice that the target type may also not exist (rules $R_{a p p-c-f a i l}$ and $R_{a p p-\lambda-f a i l}$ ), which corresponds to a case in which the $\eta$-expansion would (later) raise a cast error, and so this is what the rules $\left(R_{a p p-c-f a i l}\right)$ and $\left(R_{a p p-\lambda-f a i l}\right)$ do: for an example, consider an application such as $\left(\lambda_{\langle ? \rightarrow \text { Int }\rangle}^{\text {Int } \rightarrow \text { Int }} x .1+x\right)$ true that is well-typed -the function is cast to ? $\rightarrow$ Int and thus it can be applied to any argument- but obviously reduces to a cast error, since there is no domain in the interface that is compatible with the type of the argument.

\subsection{Soundness}

To prove the soundness of the cast language, we prove the usual lemmas, starting with subject reduction. Since we defined the compatible subtyping relation to be preserved during the evaluation and to enjoy a substitution property (see Theorem 1), the subject reduction lemma can be directly worded as follows.

Lemma 1. (Subject Reduction) - For every terms $e_{1}, e_{2} \in$ Terms ${ }^{\langle 〉}$ and every typing context $\Gamma$, if $e_{1} \mapsto e_{2}$ and $\Gamma \vdash e_{1}: \tau_{1}$, then $\Gamma \vdash e_{2}: \tau_{2}$ and $\tau_{2} \sqsubseteq \tau_{1}$.

We then prove the progress lemma, stating that every well-typed term that is not a value can be reduced. In our case, it can be reduced either to another term or to a cast error.

Lemma 2. (Progress) - For every term $e \in$ Terms $^{\langle\rangle}$, if $\emptyset \vdash e: \tau$ then $e \in$ Values $^{\langle\rangle}$or $\exists e^{\prime} \in$ Terms ${ }^{\langle\rangle}, e \mapsto e^{\prime}$ or $e \mapsto$ CastError.

Finally, the soundness of the cast language is a direct consequence of the two previous lemmas.

Theorem 2. (Soundness) - For every term $e \in$ Terms $^{\langle\rangle}$, if $\emptyset+e: \tau$ then either $e$ diverges or $\exists v \in$ Values $^{\langle\rangle}$such that $e \mapsto^{*} v$ or $e \mapsto^{*}$ CastError.

This soundness property will allow us to prove, in the next section, that the dynamic semantics of the gradually-typed lambda calculus resulting from its compilation to the cast language is sound with respect to the typing rules presented in Section 3.

\section{COMPILATION}

In this section, we define the compilation procedure of the gradually-typed lambda calculus to the cast calculus. By proving that the compilation rules map well-typed terms of the gradually-typed calculus into well-typed terms of the cast calculus and using the soundness of the latter, we can prove that the execution of compiled well-typed gradually-typed terms is sound. 


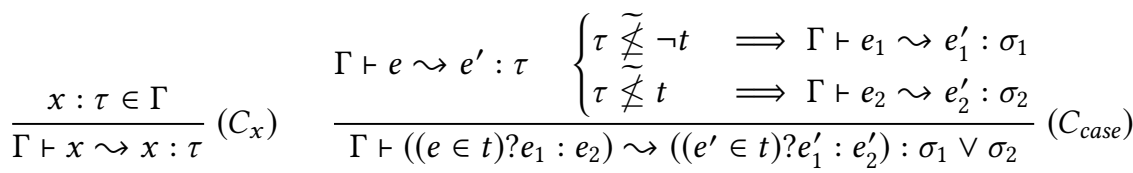

$$
\begin{aligned}
& \Gamma \vdash e_{1} \sim e_{1}^{\prime}: \tau_{1} \quad \tau_{2}^{\Uparrow} \leq \widetilde{\operatorname{dom}_{f}}\left(\tau_{1}\right) \\
& \overline{\Gamma \vdash c \leadsto c: B(c)}\left(C_{c}\right) \quad \frac{\Gamma \vdash e_{2} \leadsto e_{2}^{\prime}: \tau_{2} \quad \tau_{1}^{\Uparrow} \leq \mathbb{0} \rightarrow \mathbb{1}}{\Gamma \vdash e_{1} e_{2} \leadsto e_{1}^{\prime} e_{2}^{\prime}: \tau_{1} \widetilde{\circ} \tau_{2}}\left(C_{a p p-1}\right) \\
& \Gamma \vdash e_{1} \leadsto e_{1}^{\prime}: \tau_{1} \quad \tau_{2}^{\Uparrow} \not \leq \widetilde{\operatorname{dom}}_{f^{\prime}}\left(\tau_{1}\right) \\
& \frac{\Gamma \vdash e_{2} \leadsto e_{2}^{\prime}: \tau_{2} \quad \tau_{2}^{\Downarrow} \leq \widetilde{\operatorname{dom}}_{\rho}\left(\tau_{1}\right) \quad \tau_{1}^{\Uparrow} \leq \mathbb{0} \rightarrow \mathbb{1}}{\Gamma \vdash e_{1} e_{2} \leadsto\left\langle\tau_{1} \widetilde{\circ} \tau_{2}\right\rangle\left(e_{1}^{\prime}\left\langle\widetilde{\operatorname{dom}}_{\rho_{\rho}}\left(\tau_{1}\right)\right\rangle e_{2}^{\prime}\right): \tau_{1} \widetilde{\circ} \tau_{2}}\left(C_{a p p-2}\right)
\end{aligned}
$$

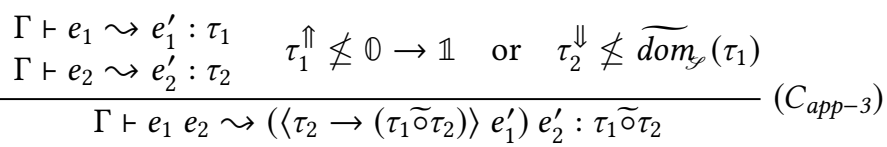

$$
\begin{aligned}
& \begin{array}{l}
\forall \sigma_{i} \rightarrow \tau_{i} \in \mathbb{I}, \\
\Gamma, x: \sigma_{i} \vdash e \leadsto e_{i}: \tau_{i}^{\prime}
\end{array} \quad e_{i}^{\prime}= \begin{cases}e_{i} & \text { if } \tau_{i}^{\prime} \sqsubseteq \tau_{i} \\
\left\langle\tau_{i}\right\rangle e_{i} & \text { otherwise }\end{cases} \\
& \Gamma \vdash \lambda^{\mathrm{I}} x . e \leadsto\left(\lambda^{\mathrm{I}} x .\left(x \in \sigma_{1}^{\Uparrow}\right) ? e_{1}^{\prime}: \cdots:\left(x \in \sigma_{i-1}^{\Uparrow}\right) ? e_{i-1}^{\prime}: e_{i}^{\prime}\right): \operatorname{TypeOf}(\mathbb{I})
\end{aligned}
$$

Fig. 4. Compilation rules for the gradually-typed language

\subsection{Compilation Rules}

The compilation rules for the gradually-typed language are presented in Figure 4. They are directed by the type system of the cast language, since we want to translate terms of the gradually-typed lambda calculus to well-typed terms of the cast language. For this reason the judgments in the rules have the form $\Gamma \vdash e \leadsto e^{\prime}: \tau$, meaning that under the typing hypothesis $\Gamma$ the term $e$ of the gradually-typed language is translated into the term $e^{\prime}$ of the cast language and that the latter has type $\tau$.

The compilation rules for constants $\left(C_{c}\right)$, variables $\left(C_{x}\right)$ and typecases $\left(C_{\text {case }}\right)$ are straightforward: the first two are the identity translation while the last one is just the component-wise translation of all sub-expressions.

There are three rules to compile applications, denoted by $\left(C_{a p p-i}\right)$. This comes from the fact that there are three possible ways to compile an application: either $(i)$ the application can be compiled "as is", or (ii) the right-hand side of the application needs to be cast to a type compatible with the type of the function, or (iii) the left-hand side needs to be cast to a function type compatible with the type of the argument.

The first case corresponds to the rule $\left(C_{a p p-1}\right)$ and is a direct consequence of the typing rules of the cast language. That is, if the two sides of the application compile respectively to $e_{1}^{\prime}$ and $e_{2}^{\prime}$ such that $e_{1}^{\prime} e_{2}^{\prime}$ is already a well-typed term of the cast language, then no cast is needed.

Otherwise, given an application $e_{1} e_{2}$ whose two sides respectively compile into $e_{1}^{\prime}: \tau_{1}$ and $e_{2}^{\prime}: \tau_{2}$ we have to decide whether to cast the function $e_{1}^{\prime}$ (to an arrow whose domain is $\tau_{2}$ ) or its argument 
$e_{2}^{\prime}$ (to the safe domain of $\tau_{1}$ ). There is one case for which no doubt is possible, namely when $\tau_{1}$ is not a function type $\left(i e, \tau_{1}^{\Uparrow} \not \leq \mathbb{O} \rightarrow \mathbb{1}\right)$. In this case we must dynamically verify that $e_{1}^{\prime}$ returns a lambda-abstraction and therefore we apply the cast to the function. This is done by the rule $\left(C_{a p p-3}\right)$. If instead $\tau_{1}$ is a function type, then we have to ensure that, at runtime, the type of the function is compatible with the type of the argument. Unfortunately there does not exist a recipe that works in all circumstances: it is not difficult to find two types $\tau_{1}$ and $\tau_{2}$ in which neither the choice of casting the argument nor the choice of casting the function is always better. ${ }^{14}$ Note however that if we cast the function, then we do it to an arrow type, while the cast of the argument does not impose any particular constraint on the type in the cast. Thus casting the function looks like a stronger requirement than casting the argument: by casting the function to an arrow we loose all the information given by the use of connectives in its type and we flatten its domain and its safe domain into a unique type. This is the reason why, in our compilation rules, we privilege to cast the argument to the safe domain of the function, but only if such a cast has a chance to succeed, that is, only if the type of the argument is a consistent subtype of the safe domain $\left(i e, \tau_{2}^{\Downarrow} \leq \widetilde{\text { dom }}_{\mathrm{g}}\left(\tau_{1}\right)\right.$ ): this is done by rule $\left(C_{a p p-2}\right)$. The condition $\tau_{2}^{\Downarrow} \leq \widetilde{\operatorname{dom}_{\rho}}\left(\tau_{1}\right)$ ensures that we will not try to apply to the argument a cast that is statically known to always fail: for instance, if we apply a function of type (Int $\rightarrow$ Int) $\wedge$ ? to an argument of type Bool it would be completely useless to cast this argument to Int, the safe domain of the function, since the cast would always fail. Thus, in this case, even though $e_{1}^{\prime}$ has a functional type, we will instead cast the function to the type Bool $\rightarrow$ ?

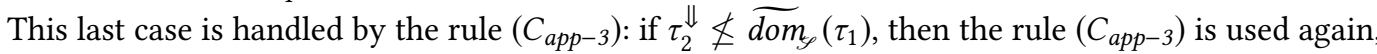
and the cast is applied to the function. Since $\tau_{2} \rightarrow\left(\tau_{1} \widetilde{\circ} \tau_{2}\right) \widetilde{\leq} \tau_{1}$, we statically know that this cast may succeed.

Finally, there remains the translation rule for lambda abstractions. Here the difficulty comes from the use of interfaces and, more generally, of intersection types. When a lambda abstraction is given an interface containing more than one type -i.e., it is typed by an intersection type-, then the function is type-checked several times, once for each type in the interface. The compilation is driven by the type system and during the typing-checking of the expression, casts are inserted to ensure soundness. The consequence of type-checking the same expression several times is that each time different casts may be added, and these may be incompatible from one pass to the other. Let us show this by an example: consider the following identity function with a bizarre but correct interface.

$$
\lambda^{\{\text {Int } \rightarrow \text { Int } ;(? \backslash \text { Int }) \rightarrow \text { Bool }\}} x . x
$$

The interface states that when the function is applied to an integer then the application returns an integer and when it is applied to any other argument then it returns a Boolean, provided it does not fail. How should we compile this function? If the function is applied to an integer, then no cast is necessary, while for all other arguments the body needs to be cast to Bool. But since this cast would be incorrect with an integer argument, the question arises whether we must insert this cast or not. The solution is to compile the body of the function by a type-case that distinguishes the two argument configurations, that is:

$$
\lambda^{\{\text {Int } \rightarrow \text { Int } ;(? \backslash \text { Int }) \rightarrow \text { Bool }\}} x .(x \in \text { Int }) ? x:\langle\text { Bool }\rangle x
$$

the body of the function is duplicated in all the branches but the cast to Bool is applied only if the argument is not of type Int.

\footnotetext{
${ }^{14}$ Consider $\tau_{1}=(($ Bool $\vee ?) \rightarrow ?) \vee(($ Int $\rightarrow$ Int $\left.) \wedge ?)\right)$ and $\tau_{2}=$ Bool $\vee ?$. The application is well-typed. However, if $e_{1}^{\prime}$ is the successor function and $e_{2}^{\prime}$ is an integer, then casting the function (to Bool $\vee ? \rightarrow$ ?) would fail while casting the argument (to the safe domain Int) would not; taking instead for $e_{1}^{\prime}$ and $e_{2}^{\prime}$ the Boolean "not" function and true would fail for a cast on the argument and succeed if the cast is on the function.
} 
This is exactly what the $\left(C_{\lambda}\right)$ rule does. In particular it transforms the body of a lambda-abstraction into a type case with as many branches as arrows in the interface, and puts into each branch the translation of the body obtained under the hypothesis that the parameter of the function has the input type of the arrow under consideration (the type case is not inserted if the interface is composed of a single arrow). Remember that, given an abstraction $\lambda^{\mathbb{I}} x$. e, its interface $\mathbb{I}=\left\{\sigma_{i} \rightarrow \tau_{i} \mid i \in I\right\}$ verifies $\forall i, j \in I, i \neq j \Longrightarrow \sigma_{i}^{\Uparrow} \wedge \sigma_{j}^{\Uparrow} \leq \mathbb{O}$ and its domain is $\widetilde{\operatorname{dom}}($ TypeOf $(\mathbb{I}))=\bigvee_{i \in I} \sigma_{i}^{\Uparrow}$. This means that the different cases of the typecase in the translation cover the whole domain of the function and are not overlapping. Thus, the order in which they are considered does not matter. As a consequence, for every value of type $\sigma \widetilde{\leq} \widetilde{\operatorname{dom}}($ TypeOf(I)), there exists a unique $i \in I$ such that $\sigma^{\Uparrow} \leq \sigma_{i}^{\Uparrow}$. This is the essence of the rule $\left(C_{\lambda}\right)$ : since the compilation of the body $e$ of the abstraction depends on the type of $x$, it checks the variable $x$ against every possible type $\sigma_{i}^{\Uparrow}$ and branches to the corresponding compiled expression $e_{i}$ (where $e \leadsto e_{i}$ under the hypothesis that $x$ has type $\sigma_{i}$ ). Finally, to ensure that the compiled function is well-typed (in the type system of the cast language) with respect to its interface, the rule also add casts to branches that require it. For instance, in our example in (4) no cast is inserted in the first branch (since if $x$ : Int then the body has type Int which is exactly the type in the interface) but a cast is inserted in the second (since $x$, and thus the body, has type ? \Int and ? \Int $\nsubseteq$ Bool).

\subsection{Safety and Soundness}

The compilation procedure compiles gradually-typed terms to terms of the cast language, and thus implicitly defines the reduction semantics of the gradually-typed language. The safety property for this language states that the compilation of every well-typed term reduces to a value, a cast error, or diverges. However, for this statement to be meaningful, the compilation must be exhaustive; that is, it should be possible to compile every well-typed term of the gradually-typed language. This is formally stated as follows.

Lemma 3. (Exhaustiveness of Compilation) - For every term $e \in$ Terms and every typing context $\Gamma$, if $\Gamma \vdash e: \tau$ then $\Gamma \vdash e \leadsto e^{\prime}: \tau$ where $e^{\prime} \in$ Terms $^{\langle\rangle}$.

Moreover, we can prove that the compilation procedure preserves the type of the compiled term.

Lemma 4. (Type Preservation by Compilation) - For every term e $\in$ Terms and every typing context $\Gamma$, if $\Gamma \vdash e \leadsto e^{\prime}: \tau$ then $\Gamma \vdash e^{\prime}: \tau$.

These two lemmas can be summarized as the following theorem, proving the soundness of the compilation procedure with respect to both type systems.

Theorem 3. (Soundness of Compilation) - For every term $e \in$ Terms and every typing context $\Gamma$, if $\Gamma \vdash e: \tau$ then $\Gamma \vdash e \leadsto e^{\prime}: \tau$, where $e^{\prime} \in$ Terms $^{\langle\rangle}$and $\Gamma \vdash e^{\prime}: \tau$.

The safety property of the cast language stated in Theorem 2 can then be "lifted" to the graduallytyped lambda calculus.

Corollary 1. (Safety of the Gradually-Typed Language) - For every term e $\in$ Terms, if $\emptyset \vdash e: \tau$, then $e \leadsto e^{\prime}: \tau$ where $e^{\prime} \in$ Terms ${ }^{\langle\rangle}$and either $e^{\prime}$ diverges, or $\exists v \in$ Values ${ }^{\langle\rangle}$such that $e^{\prime} \mapsto^{*} v$ and $\emptyset \vdash v: \tau^{\prime} \widetilde{\leq} \tau$, or $e^{\prime} \mapsto^{*}$ CastError.

\section{CONCLUSION}

In this work, we presented a foundational study for gradually-typed languages with union and intersection types and argued that the combination of the former with the latter allows a smoother and finer-grained transition between static and dynamic typing, than what is possible with the 
current state of the art. As the use of the unknown type "?" allows the programmer to gradually add type information in selected parts of the program, so the use of unions and intersections allows the same programmer to gradually refine the type information about a given part of the program or a single expression, thus enabling a new style of gradual programming. While adding unions and intersections inside arrows brings real benefits at no cost, we have also seen that for adding intersections on arrows there is instead a price to pay: intersections of arrows allow the programmer to write overloaded functions, but when these are combined with gradual types their code needs to be specialized to insert casts that depend on the type of the argument. This happens both in the semantics of the cast language (where the cast to apply to the argument is different in the case of interfaces with multiple arrows) and in the compilation rules (where the body of a $\lambda$-abstraction is compiled into a type-case when the interface contains multiple arrows). Further research is needed to study how to alleviate this cost.

The language presented in this work is minimalist and if we want our study to scale to real world functional languages, then it is necessary to extend it in several directions, foremost by adding advanced features, such as pattern matching, type recursion, and new type constructors (in particular, products and records). Equally important will be the addition of polymorphic types, an addition that we plan to pursue by drawing ideas from both the work on polymorphic set-theoretic types by Castagna et al. [2015, 2014] and the work on polymorphic gradual types by Siek and Vachharajani [2008] and Garcia and Cimini [2015]. This work will be followed by the study of some forms of type reconstruction and local type inference on the basis of what was already done on set-theoretic types by Castagna et al. [2016]. Only then we will be able to see whether and how the techniques whose study started here apply to languages such as JavaScript and how they compare and/or whether they may bring any contribution to approaches such as the one of the language Flow that we described in Section 1.3 on related work.

From a more theoretical point of view, we saw that using type connectives increases the discretionary of adding casts in an application. Our choice, embodied by the compilation rule $\left(C_{a p p-2}\right)$, was to push casts on the argument part of an application as much as possible. However, it may be worth exploring whether by a "threesome" approach as proposed by Siek and Wadler [2010] one could find more expressive intermediate solutions. Another property that may also be worth verifying for our system is the gradual guarantee [Siek et al. 2015], which states that a program that runs without errors still does with less precise type annotations.

We completely disregarded error-message generation, both at static and at dynamic time. In particular, we did not considered the so-called blame. As pointed out by an anonymous referee, a "blame theorem", as presented by Wadler and Findler [2009], is not worth proving for our calculus, since the wrong casts might be blamed. The reason for that lies in the fact that we chose to collapse casts on functions (see rule $R_{\text {cast }-\lambda}$ in Figure 3 ). While this choice makes the calculus simpler without hindering soundness, it yields a formalism unfit to finger culprits. In particular, complete monitoring [Dimoulas et al. 2012] fails for a calculus that omit casts in this way since it cannot keep track of every owner of a term (see Dimoulas et al. [2012] for a general discussion). We are currently studying a different cast language that will make the study of blame and error-message generation possible, so that whenever the execution of a program results in a cast error, then it is the dynamically-typed part of the program that must be blamed for that. Finally, we would like to explore how this study applies when one tries to extend it with generalized abstract data types.

\section{ACKNOWLEDGMENTS}

The authors would like to thank Tommaso Petrucciani, Jeremy Siek, Éric Tanter, and the anonymous reviewers for their remarks and suggestions which allowed us to significantly improve this work. 


\section{REFERENCES}

Véronique Benzaken, Giuseppe Castagna, and Alain Frisch. 2003. CDuce: an XML-centric general-purpose language. In Proceedings of the 8th ACM SIGPLAN International Conference on Functional Programming (ICFP '03). ACM, 51-63.

Giuseppe Castagna. 2015. Covariance and Contravariance: a fresh look at an old issue (a primer in advanced type systems for learning functional programmers). (2015). Unpublished manuscript, available at the author's web page.

Giuseppe Castagna, Giorgio Ghelli, and Giuseppe Longo. 1995. A calculus for overloaded functions with subtyping Information and Computation 117, 1 (1995), 115-135.

Giuseppe Castagna, Kim Nguyen, Zhiwu Xu, and Pietro Abate. 2015. Polymorphic Functions with Set-Theoretic Types. Part 2: Local Type Inference and Type Reconstruction. In Proceedings of the 42nd ACM Symposium on Principles of Programming Languages (POPL '15). ACM, 289-302.

Giuseppe Castagna, Kim Nguyen, Zhiwu Xu, Hyeonseung Im, Sergueï Lenglet, and Luca Padovani. 2014. Polymorphic Functions with Set-Theoretic Types. Part 1: Syntax, Semantics, and Evaluation. In Proceedings of the 41st ACM Symposium on Principles of Programming Languages (POPL '14). ACM, 5-17.

Giuseppe Castagna, Tommaso Petrucciani, and Kim Nguyen. 2016. Set-Theoretic Types for Polymorphic Variants. In Proceedings of the 21st ACM SIGPLAN International Conference on Functional Programming (ICFP '16). ACM, 378-391.

Avik Chaudhuri. 2014. Flow: A static type checker for favaScript. Facebook. https://flowtype.org.

Patrick Cousot and Radhia Cousot. 1977. Abstract interpretation: a unified lattice model for static analysis of programs by construction or approximation of fixpoints. In Proceedings of the 4th ACM SIGACT-SIGPLAN Symposium on Principles of Programming Languages (POPL '77). ACM, 238-252.

Christos Dimoulas, Sam Tobin-Hochstadt, and Matthias Felleisen. 2012. Complete Monitors for Behavioral Contracts. In Programming Languages and Systems - 21st European Symposium on Programming, ESOP'12. 214-233.

Alain Frisch. 2004. Regular Tree Language Recognition with Static Information. In IFIP 18th World Computer Congress TC1, 3rd International Conference on Theoretical Computer Science (TCS2004) (IFIP), Vol. 155. Kluwer/Springer, 661-674.

Alain Frisch, Giuseppe Castagna, and Véronique Benzaken. 2008. Semantic Subtyping: dealing set-theoretically with function, union, intersection, and negation types. F. ACM 55, 4 (2008), 1-64.

Ronald Garcia and Matteo Cimini. 2015. Principal Type Schemes for Gradual Programs. In Proceedings of the 42nd Annual ACM SIGPLAN-SIGACT Symposium on Principles of Programming Languages (POPL '15). ACM, 303-315.

Ronald Garcia, Alison M Clark, and Éric Tanter. 2016. Abstracting gradual typing. In Proceedings of the 43rd Annual ACM SIGPLAN-SIGACT Symposium on Principles of Programming Languages (POPL '16). ACM, 429-442.

Khurram A. Jafery and Joshua Dunfield. 2017. Sums of Uncertainty: Refinements Go Gradual. In Proceedings of the 44th ACM SIGPLAN Symposium on Principles of Programming Languages (POPL '17). ACM, 804-817.

Nico Lehmann and Éric Tanter. 2017. Gradual refinement types. In Proceedings of the 44th ACM SIGPLAN Symposium on Principles of Programming Languages (POPL '17). ACM, 18-20.

John C. Reynolds. 1996. Design of the Programming Language Forsythe. Technical Report CMU-CS-96-146. Carnegie Mellon University.

Jeremy G. Siek and Walid Taha. 2006. Gradual Typing for Functional Languages. In Proceedings of Scheme and Functional Programming Workshop. ACM, 81-92.

Jeremy G. Siek and Sam Tobin-Hochstadt. 2016. The Recursive Union of Some Gradual Types. In A List of Successes That Can Change the World: Essays Dedicated to Philip Wadler on the Occasion of His 60th Birthday, Sam Lindley, Conor McBride, Phil Trinder, and Don Sannella (Eds.). Springer, 388-410.

Jeremy G. Siek and Manish Vachharajani. 2008. Gradual typing with unification-based inference. In Proceedings of the 2008 Symposium on Dynamic languages. ACM, 7.

Jeremy G. Siek, Michael M Vitousek, Matteo Cimini, and John Tang Boyland. 2015. Refined criteria for gradual typing. In LIPIcs-Leibniz International Proceedings in Informatics, Vol. 32. Schloss Dagstuhl-Leibniz-Zentrum fuer Informatik.

Jeremy G. Siek and Philip Wadler. 2010. Threesomes, with and Without Blame. In Proceedings of the 37th Annual ACM SIGPLAN-SIGACT Symposium on Principles of Programming Languages (POPL '10). ACM, 365-376.

Asumu Takikawa, Daniel Feltey, Ben Greenman, Max S. New, Jan Vitek, and Matthias Felleisen. 2016. Is Sound Gradual Typing Dead?. In Proceedings of the 43rd Annual ACM SIGPLAN-SIGACT Symposium on Principles of Programming Languages (POPL '16). ACM, 456-468.

Sam Tobin-Hochstadt and Matthias Felleisen. 2008. The Design and Implementation of Typed Scheme. In Proceedings of the 35th Annual ACM SIGPLAN-SIGACT Symposium on Principles of Programming Languages (POPL '08). ACM, 395-406.

Philip Wadler and Robert Bruce Findler. 2009. Well-Typed Programs Can'T Be Blamed. In Proceedings of the 18th European Symposium on Programming Languages and Systems (LNCS). Springer, 1-16.

Andrew K. Wright and Matthias Felleisen. 1994. A Syntactic Approach to Type Soundness. Information and Computation 115, 1 (1994), $38-94$

Proc. ACM Program. Lang., Vol. 1, No. ICFP, Article 41. Publication date: September 2017. 